\title{
Constraints on Extrasolar Planet Populations from VLT NACO/SDI and MMT SDI and Direct Adaptive Optics Imaging Surveys: Giant Planets are Rare at Large Separations
}

\author{
Eric L. Nielsen ${ }^{1}$, Laird M. Close, and Beth A. Biller \\ Steward Observatory, University of Arizona, Tucson, AZ 85721 \\ enielsen@as. arizona.edu \\ and \\ Elena Masciadri \\ INAF-Osservatorio Astrofisico di Arcetri, Italy
}

\begin{abstract}
We examine the implications for the distribution of extrasolar planets based on the null results from two of the largest direct imaging surveys published to date. Combining the measured contrast curves from 23 of the stars observed with the VLT NACO adaptive optics system by Masciadri et al. (2005), and 47 of the stars observed with the VLT NACO SDI and MMT SDI devices by Biller et al. (2007) (for a total of 59 unique stars: the median star for our survey is a $30 \mathrm{Myr}$ $\mathrm{K} 2$ star at $25 \mathrm{pc}$ ), we consider what distributions of planet masses and semimajor axes can be ruled out by these data, based on Monte Carlo simulations of planet populations. We can set the following upper limit with $95 \%$ confidence: the fraction of stars with planets with semi-major axis between 15 and $100 \mathrm{AU}$, and mass above $4 \mathrm{M}_{J u p}$, is $20 \%$ or less. Also at the $95 \%$ confidence level, with a distribution of planet mass of $\frac{d N}{d M} \propto M^{-1.16}$ in the range of $0.5-13 \mathrm{M}_{\text {Jup }}$, we can rule out a power-law distribution for semi-major axis $\left(\frac{d N}{d a} \propto a^{\alpha}\right)$ with index 0 and upper cut-off of $16 \mathrm{AU}$, and index -0.5 with an upper cut-off of $42 \mathrm{AU}$. For the distribution suggested by Cumming et al. (2007), a power-law of index -0.61 , we can place an upper limit of $70 \mathrm{AU}$ on the semi-major axis distribution, again at the $95 \%$ confidence level. In other words, given these assumptions for the semi-major axis distribution, and using the models of Burrows et al. (2003), giant planets are rare past $70 \mathrm{AU}$. At the $68 \%$ confidence level, these upper limits
\end{abstract}

\footnotetext{
${ }^{1}$ Michelson Fellow
} 
state that fewer than $8 \%$ of stars have a $>4 \mathrm{M}_{J u p}$ planet between 15 and 100 $\mathrm{AU}$, and a power-law distribution for semi-major axis with index $0,-0.5$, and -0.61 cannot have giant planets beyond 11,20 , and 27 AU, respectively. With our current observations, we cannot reject the models of Ida \& Lin (2004) for the masses and semi-major axes of giant planets with better than $50 \%$ confidence. In general, we find that even null results from direct imaging surveys are very powerful in constraining the distributions of giant planets at large separations, but more work needs to be done to close the gap between planets that can be detected by direct imaging, and those to which the radial velocity method is sensitive.

Subject headings: stars: planetary systems

\section{Introduction}

There are currently well over 200 known extrasolar planets, the bulk of which were discovered by radial velocity surveys (e.g. Butler et al. (2006)). While this field has initially been dominated by the study of the relatively easy-to-find Hot Jupiters (planets with orbital periods of order days), over the past several years there has been an increasing amount of data describing planets in larger orbits. In particular, Fischer \& Valenti (2005) compared radial velocity target stars with known planets to stars that had been monitored but did not show signs of planets; they concluded that about $5 \%$ of stars had planets of mass greater than $1.6 M_{J u p}$, in orbits shorter than 4 years (within $2.5 \mathrm{AU}$ ). Additionally, they determined that planet fraction increased with the host star's metal abundance. Butler et al. (2006) have also considered the distributions of semi-major axis and planet mass of known radial velocity planets, and found that both distributions are well-fit by power laws. Cumming et al. (2007) have examined the biases of the radial velocity technique, and found that the semi-major

axis distribution found by Butler et al. (2006), $\frac{d N}{d P} \propto P^{-1}$, should be modified in light of the decreasing sensitivity of the radial velocity method with orbital distance, and suggest a power law index of -0.74 for period, instead (for solar-like stars, this corresponds to a power law distribution for semi-major axis where $\left.\frac{d N}{d a} \propto a^{-0.61}\right)$.

One outstanding question is how the abundance of planets varies as one considers planets in longer orbits. Raymond (2006) has studied the dynamics of terrestrial planet formation in systems with giant planets, and found from numerical simulations that giant planets impede the formation of earth-like planets when the giant planet orbits within $2.5 \mathrm{AU}$, and that water delivery to the planet is only possible in significant amounts when the giant planet is beyond 3.5 AU. The full extent to which giant planets impede (or encourage) terrestrial 
planet formation is still unknown. A greater understanding of the distribution of giant planets is a precursor to investigating the conditions under which terrestrial planets form and evolve.

The global distribution of giant planets has also been considered from the theoretical direction. Ida \& Lin (2004) have produced distributions of planets forming in disks by core accretion, showing a continuation of a power law from the radial velocity regime (within 2.5 AU) for giant planets, out to about $10 \mathrm{AU}$, then trailing off at larger radii. It is possible that the lack of outer planets in these simulations may be due (at least in part) to the fact that these models do not consider the effects of planet-planet scattering after planets are formed. In order to constrain such models it is necessary to measure the distribution of giant planets in longer orbits, so as to fully sample parameter space.

With the advent of adaptive optics systems on large $(\sim 8 \mathrm{~m})$ telescopes, the ability to detect and characterize planets by directly imaging the companion is becoming increasingly viable. Already planetary mass companions (in most cases $\sim 13 \mathrm{M}_{\text {Jup }}$ at $40-300$ AU, or even lower mass objects with brown dwarf hosts) have been detected in certain favorable circumstances (e.g. companions to 2MASS1207: Chauvin et al. (2004), AB Pic: Chauvin et al. (2005), Oph 1622: Brandeker et al. (2006), Luhman et al. (2007), Close et al. (2007b), CHXR 73: Luhman et al. (2006), and DH Tau: Itoh et al. (2005)), and numerous surveys are underway for planets around nearby, young stars (since a self-luminous planet is brightest at young ages). While the paucity of traditional planets (that is, planets $<13$ $\mathrm{M}_{\text {Jup }}$ and $<40 \mathrm{AU}$ orbiting a star) detected by this method has been disappointing, in this paper we consider how even a null result from these direct imaging surveys can be used to set constraints on the population of giant planets. As the sensitivity of radial velocity surveys to planets at larger separations decreases (due both to the smaller radial velocity signal, and the longer orbital period, requiring a longer time baseline of observations to adequately constrain the orbital parameters), at orbits wider than 10 AU only direct imaging is efficient at characterizing the extrasolar planet population.

Previous work has been done by Masciadri et al. (2005) to study this region of parameter space with a survey of 28 young, nearby stars, with a null result for planets. Masciadri et al. (2005) found that their observations were sufficient to detect a $5 \mathrm{M}_{\text {Jup }}$ planet at projected separations greater than $14 \mathrm{AU}$ around 14 of their target stars, and above $65 \mathrm{AU}$ for all 28 stars. Similarly, their observations would have been sensitive to a $10 \mathrm{M}_{\text {Jup }}$ planet with a projected separation of 8.5 AU or beyond for half their sample, and greater than $36 \mathrm{AU}$ for the full sample. These results (obtained by adopting published ages for the target stars, and using the appropriate planet models of Baraffe et al. (2003)) point to a rarity of giant planets at large separations from their parent stars. In this paper we enlarge the sample by 
a factor of 3, and consider the implications of our null result with respect to the full orbital parameters of potential planets. We aim to set quantitative limits on the distribution of planets in semi-major axis space, and statistically rule out models of planet populations.

\section{Observations}

We begin with contrast plots (sensitivity to faint companions as a function of angular separation from the target star) from two surveys for extrasolar planets, using large telescopes and adaptive optics. Masciadri et al. (2005) carried out a survey of 28 young, nearby, late-type stars with the NACO adaptive optics system at the 8.2 meter Very Large Telescope (VLT). These observations have exposure times of order 30 minutes, with stars being observed in the $\mathrm{H}$ or $\mathrm{Ks}$ bands. Subsequent to these observations, a survey of 54 young, nearby stars of a variety of spectral types (between A and M) was conducted between 2003 and 2005, with the results reported in Biller et al. (2007). This second survey used the Simultaneous Differential Imager (SDI) at the 6.5 meter MMT and the VLT, an adaptive optics observational mode that allows higher contrasts by imaging simultaneously in narrow wavelength regions surrounding the $1.6 \mu \mathrm{m}$ methane feature seen in cool brown dwarfs and expected in extrasolar planets (Lenzen et al. 2004; Close et al. 2005). This allows the light from a hypothetical companion planet to be more easily distinguishable from the speckle noise floor (uncorrected starlight), as the two will have very different spectral signatures in this region. This translates to higher sensitivity at smaller separation than the observations of Masciadri et al. (2005), which was conducted before the VLT SDI device was commissioned (see Fig. 14 of Biller et al. (2007) for a more detailed comparison of the two surveys). For most of these SDI targets, the star was observed for a total of 40 minutes of integration time, which includes a 33 degree roll in the telescope's rotation angle, in order to separate speckles-which are created within the instrument, and so will not rotate-from a physical companion, which will rotate on the sky (Biller et al. 2006).

For both sets of target stars, contrast curves have been produced which give the $5 \sigma$ noise in the final images as a function of radius from the target stars, and thus an upper limit on the flux of an unseen planet in the given filter of the observations. As no planets were detected in either survey at the $5 \sigma$ level, we use these contrast curves to set upper limits on the population of extrasolar planets around young, nearby stars. 


\section{1. $\quad$ Target Stars}

We construct a target list using 13 stars from the Masciadri et al. (2005) survey, and 46 stars from the survey of Biller et al. (2007), for a total of 59 targets This first cut was made by considering stars from the two surveys that had contrast curves, and stars whose age could be determined by at least one of: group membership, lithium abundance, and the activity indicator $\mathrm{R}_{H K}^{\prime}$ (in three cases, ages from the literature were used, though these are stars that are generally older than our sample as a whole, and so uncertainties in the assumed ages will not adversely affect our results). Ages are determined by taking the age of the moving group to which the target star belongs; if the star does not belong to a group, the lithium or $\mathrm{R}_{H K}$ age is used, or the two are averaged if both are available. Lithium ages are found by comparing to lithium abundances of members of clusters of known ages, and similarly for $\mathrm{R}_{H K}$ (Mamajek 2007). We give the full target list in Table 1, and details on the age determination in Table 2, We also plot our target stars in Fig. 1, Overall, our mean survey object is a 30 Myr K2 star at 25 pc.

\section{Monte Carlo Simulations}

In order to place constraints on the properties of planets from our null results, we run a series of Monte Carlo simulations of an ensemble of extrasolar planets around each target star. Each simulated planet is given full orbital parameters, an instantaneous orbital phase, and amass, then the planet's magnitude in the observational band is determined from these properties (using the target star's age and distance, and theoretical mass-luminosity relations) as is its projected separation from the star. Finally, this magnitude is compared to the measured contrast curve to see if such a planet could be detected. Determining which simulated planets were detected, and which were not, allows us to interpret the null result in terms of what models of extrasolar planet populations are excluded by our survey results.

\subsection{Completeness Plots}

As in Biller et al. (2007), we use completeness plots to illustrate the sensitivity to planets as a function of planet mass and semi-major axis. To do this, for each target star, we create a grid of semi-major axis and planet mass. At each grid location we simulate $10^{4}$ planets, and then compute what fraction could be detected with the contrast curve for that star.

In general, most orbital parameters are given by well-known distributions. Inclination angle has a constant distribution in $\sin (\mathrm{i})$, while the longitude of the ascending node and the 
mean anomaly are given by uniform distributions between 0 and $2 \pi$. Since contrast plots are given in terms of radius alone, it is not necessary to consider the argument of periastron in the simulations.

To simulate the eccentricities of the planet orbits, we examine the orbital parameters of known extrasolar planets from radial velocity surveys. We consider the orbits of planets given by Butler et al. (2006), and show their distribution of eccentricities in Fig. 2. By dividing the sample into two populations, based on a cut at an orbital period of 21 days, we can separate out the population of Hot Jupiters, which we expect to have experienced orbital circularization as a result of their proximity to their host stars. For both sets of populations, we fit a simple straight line to the distributions (the logarithmic bins for the Hot Jupiter population means this line translates to a quadratic fit). We note that the Hot Jupiter fit is plagued by small number statistics, and so the fit is likely to be less reliable than that for long period planets. Even for our closest target stars, such an orbital period gives star-planet separations less than 0.1 ", a regime where our contrast curves show we are not sensitive to planets. As a result, the manner in which the orbits of hot Jupiters are simulated has effectively no impact on our final results.

For each simulated planet, the on-sky separation is determined at the given orbital phase, and mass is converted into absolute $\mathrm{H}$ or $\mathrm{Ks}$ magnitude, following the mass-luminosity relations of both Burrows et al. (2003) or Baraffe et al. (2003). In the case of the models of Burrows et al. (2003), we use a Vega spectrum to convert the various model spectra into absolute $\mathrm{H}$ and Ks magnitudes. We also note that these models only cover a range of planet masses greater than $1 M_{\text {Jup }}$, and ages above $100 \mathrm{Myr}$. Since the range of ages of our target stars extends down to $10 \mathrm{Myr}$, and we wish to consider planets down to masses of $0.5 \mathrm{M}_{\text {Jup }}$, we perform a simple extrapolation of the magnitudes to these lower ages and masses. While this solution is clearly not ideal, and will not reflect the complicated physical changes in these objects as a function of mass and age, we feel that this method provides a good estimation of how the Burrows et al. (2003) models apply to our survey.

At this point, we use the distance to the target star, as well as its 2MASS flux in either $\mathrm{H}$ or $\mathrm{Ks}$, to find the delta-magnitude of each simulated planet. With this, and the projected separation in the plane of the sky, we can compare each simulated planet to the $5 \sigma$ contrast curve, and determine which planets can be detected, and which cannot. We also apply a minimum flux limit, based on the exposure time of the observation, as to what apparent magnitude for a planet is required for it to be detected, regardless of its distance from the parent star. For the SDI observations, which make use of optimized (compared to basic H-band observing) methane filters, we add an additional factor of $\Delta H=0.6$ magnitudes (appropriate for a T6 spectral type, a conservative estimate for young planets; see Biller et al. 
(2007) for details on this factor). Also, for these SDI observations, we place an upper cut-off on masses where, for the age of the system, the planet reaches an effective temperature of $1400 \mathrm{~K}$ (we use Burrows et al. (2003) to give the mass of this cut-off as a function of age). Above this temperature, methane in the atmosphere of the planet is destroyed, and the methane feature disappears, so that the SDI subtraction now attenuates any planets, as well as stellar speckles. While non-methane objects further out than 0.2 " are not totally removed in the image (e.g. Fig. 4 of Nielsen et al. (2005)), for consistency we ignore this possibility when considering upper limits.

We plot an example of this simulation at a single grid point in mass and semi-major axis in Fig. 3. for the target star GJ 182, the 18th best target star in our survey, using the planet models of Burrows et al. (2003). $10^{4}$ simulated planets (only 300 are plotted in this figure, for clarity) are given a single value of mass $\left(4.5 M_{J u p}\right)$ and semi-major axis (10 AU). Since each planet has unique orbital parameters (eccentricity, viewing angle, and orbital phase), the projected separation varies from planet to planet, so some are above the $5 \sigma$ detection threshold of the contrast curve (the blue dots in the figure), while others are not (the red dots). For this particular target star and simulated planets of mass $4.5 \mathrm{M}_{\text {Jup }}$ and semi-major axis $10 \mathrm{AU}, 13 \%$ of these planets can be detected.

To produce a complete contour plot, we consider a full grid of mass (100 points, between 0.5 and $17 M_{J u p}$ ) and semi-major axis (again 100 points, between 1 and $210 \mathrm{AU}$ ), running

a simulation as in Fig. 3 at each of the 100,000 grid points. We then plot contours showing what fraction of planets we can detect that have a given mass and semi-major axis, as in Fig. 4, again for the target star GJ 182. The hard upper limit is set by the methane cut-off, where the planet mass becomes high enough (for the age of the given target star) for the effective temperature to exceed $1400 \mathrm{~K}$, at which point the methane feature is much less prominent in the planet's spectrum. The fact that the contours do not precisely line up at this limit is simply a result of the interpolation used to plot the contours. If GJ 182 had a planet with mass and semi-major axis such that it would fall within the innermost contour, we would have an $80 \%$ chance of detecting it. Obviously, these plots make no statements about whether these stars have planets of the given parameters, but instead simply express our chances of detecting such a planet if it did exist.

\subsection{Detection Probabilities Given an Assumed Distribution of Mass and Semi-major Axis of Extrasolar Planets}

With the large number of currently-known extrasolar planets, it is possible to assume simple power-law representations of the distributions of mass and semi-major axis of giant 
planets, which allows for a more numerical interpretation of our null result. Butler et al. (2006) suggest a power laws of the form $\frac{d N}{d M} \propto M^{-1.16}$ for mass, while Cumming et al. (2007) use the power law $\frac{d N}{d a} \propto a^{-0.61}$ for semi-major axis, in order to describe the distributions of known extrasolar planets. We make histograms for mass (Fig. [5) and semi-major axis (Fig. 6) from the parameters of all currently-known extrasolar planets (parameters taken from the Catalog of Nearby Exoplanets, http://exoplanets.org, in May 2007). In both cases, the power laws do a reasonable job of fitting the data, above $1.6 M_{\text {Jup }}$ and within 3.5 AU. For smaller planets, or longer periods, we would expect the observational biases of the radial velocity method to make the sample incomplete, thus accounting for the drop-off of planets from what would be predicted by the power law. We echo the caution of Butler et al. (2006) that these planets are drawn from many inhomogeneous samples, but we believe with the relatively large numbers, the derived distributions are not far off from the actual distributions.

In general, then, if one assumes that these power laws are universal to all stars, and that the semi-major axis power law continues to larger separations with the same index, the only outstanding question is to what outer limit (or "cut-off") this distribution continues before it is truncated. This cut-off is a term that can be uniquely well constrained by our survey. We return to this issue, after considering the results from our survey, in Section 4.3.

For the Monte Carlo simulations using these assumptions, in addition to the other orbital parameters, we obtain mass and semi-major axis through random variables that follow the given power law distributions, and again find what fraction of planets can be detected given the contrast curve for that particular target star. An example of this simulation, again for GJ 182, is given in Fig. 7, showing that with an assumed upper limit for semi-major axis of $70 \mathrm{AU}$, and a power law with index -0.61, and mass power law index of -1.16 between 0.5 and $13 \mathrm{M}_{J u p}$, we would be able to detect $17 \%$ of the simulated planets. Again, for this figure, we simply show the results using the models of Burrows et al. (2003).

\section{Analysis}

Having developed the tools to produce completeness plots, as well as compute the fraction of detected planets for various assumed models of semi-major axis, we proceed to combine the results over all our target stars in order to place constraints on the populations of extrasolar planets from these two surveys. 


\subsection{Planet Fraction}

A simplistic description of the number of planets expected to be detected is given by the expression

$$
N(a, M)=\sum_{i=1}^{N_{o b s}=59} f_{p}(a, M) P_{i}(a, M)
$$

That is, the number of planets one expects to detect at a certain semi-major axis and mass is given by the product of the detection probability $\left(P_{i}\right)$ for a planet of that mass $(\mathrm{M})$ and semi-major axis $(\mathrm{a})$, and the fraction of stars $\left(f_{p}\right)$ that contain such a planet (or "planet fraction"), summed over all target stars. In this treatment, we ignore two major effects: we assume that there is no change in the mass or separation distribution of planets, or their overall frequency, as a function of spectral type of the primary; we also do not consider any metallicity dependence on the planet fraction. While these assumptions are clearly incorrect (e.g. Johnson et al. (2007), Fischer \& Valenti (2005)), it is a good starting point for considering what constraints can be placed on the population of extrasolar planets. Also, we note that our sample includes 24 binaries, which may inhibit planet formation, though most of these binaries have separations greater than 200 AU. This leaves only ten binaries with separations in the range of likely planet orbits that might potentially contaminate our results. For simplicity, we leave these binaries in our sample, and we will return to this issue in Section 4.3.

Using the contrast curves from each of our 59 targets stars (as in Fig. 4), we simply sum the fraction of detected planets at each grid points for all of our stars. This gives the predicted number of detected planets at each combination of mass and semi-major axis, assuming each target star has one planet of that mass and semi-major axis $\left(f_{p}(a, M)=1\right)$.

More instructively, if we assume a uniform value of the planet fraction for all target stars, we can solve for $f_{p}$. Then by setting the predicted number of planets equal to three $\left(\Sigma P_{i}=3\right)$, our null result allows us to place an upper limit on the planet fraction at the $95 \%$ confidence level, since our survey measured a value of $\mathrm{N}(\mathrm{a}, \mathrm{M})=0$ (in a Poisson distribution, the probability of obtaining a certain value is given by $P=e^{-\mu} \frac{\mu^{\nu}}{\nu !}$, which for the case of a null result, $\nu=0$, becomes $P=e^{-\mu}$. So a $95 \%$ confidence level requires an expectation value, $\mu$, of 3 planets). We can thus rewrite Eq. 1 as

$$
f_{p}(a, M) \leq \frac{3}{\sum_{i=0}^{N_{o b s}} P_{i}(a, M)}
$$

Put another way, if we expected, from our $5 \sigma$ contrast curves, to detect 12 planets $(\Sigma \mathrm{P}=12$, for $\left.f_{p}=1\right)$, in order to have actually detected 0 planets from our entire survey $(\mathrm{N}<1)$, the 
planet fraction must be less than $25 \%\left(f_{p}<0.25\right)$, at the $95 \%$ confidence level. Doing this at each point in the grid of our completeness plots allows for an upper limit on the planet fraction as a function of mass and semi-major axis.

We plot the contours of this upper limit in Fig. 8, using the planet models of Burrows et al. (2003). The sharp inward jags in the shape are the result of SDI targets losing planets beyond the methane cut-off, for masses set by the age of the various moving groups. A general result from these data is that, again at the $95 \%$ confdience level, we would expect fewer than $20 \%$ of stars to have planets of mass greater than $4 M_{J u p}$ with semi-major axis between 15 and $100 \mathrm{AU}$. There appears to be no oasis of giant planets (more massive than Jupiter) in long-period orbits: at the $85 \%$ confidence level, this upper limit on the fraction of stars with giant planets drops to less than $10 \%$.

We present the same plot, this time using the COND models of Baraffe et al. (2003), in Fig. 9. The main difference between the two sets of models is a systematic offset to higher masses for the same age and NIR magnitudes when moving to the COND models. Marley et al. (2007) have recently produced a third set of models, which globally predict lower luminosities for giant planets. Since synthetic spectra for these models are not currently available, we do not examine the consequences of these models here, though we discuss possible effects in Section 5. But we note that while at $30 \mathrm{Myr}$ and at $4 \mathrm{M}_{\text {Jup }}$ there is only a $\sim 3 \mathrm{X}$ decrease in the luminosity predicted by Marley et al. (2007) compared to Burrows et al. (2003), the temperature of these objectss is lower, therefore increasing the number of planets with methane that can be detected using SDI. As a result, even with the future use of the Marley et al. (2007) modes, our results will not change dramatically, with respect to the total number of planets to which we are sensitive.

\subsection{Host Star Spectral Type Effects}

From the perspective of direct imaging searches for extrasolar planets, M-stars are especially appealing: their lower intrinsic luminosity means a given achievable contrast ratio allows fainter companions to be detected, and so makes the detection of planet-mass companions seem more likely. Nevertheless, there appears to be mounting evidence that even if the fraction of stars with planets does not decline when moving to later spectral types (as suggested by Johnson et al. (2007)), the mean planet mass is likely to decrease (e.g. Butler et al. (2004), Bonfils et al. (2005)). While it seems natural that the initial mass of the circumstantial disk (and so the mass of formed planets) should scale with the mass of the parent star, as of yet there is not sufficient data to quantify this relation. Additionally, it is problematic for us to model planet distributions on radial velocity planets, when these 
planets are almost entirely in systems with a host star of spectral type F, G, or K.

In order to investigate this effect, we divide our stars by spectral type, then recompute what limits we can set on the planet fraction. In Fig. 10 and 11 we plot the upper limit on the planet fraction (again at 95\% confidence) for only the solar-like stars ( $\mathrm{K}$ or earlier) in our survey (44 of our 59 target stars, this includes the one A star in our survey, HD 172555 A). As we would expect, the statistics in the inner contour remain largely the same, but the contours move upward and to the left, as less massive and closer-in planets become harder to detect against the glare of earlier-type stars.

We also consider the fifteen $M$ stars in our sample, in Fig. 12 and 13. The effect of the smaller number of stars is apparent, though the shape of the contours is again roughly the same. If, as is suggested by Johnson et al. (2007), planets are less common around low mass stars, or less massive stars harbor less massive planets, it becomes difficult to probe the population of $\mathrm{M}$ star planets with surveys such as these.

\subsection{Semi-Major Axis Distribution}

We now consider what constraints can be placed on planet populations if we assume a basic form to the distributions. In particular, if we take the mass power-law from currentlyknown extrasolar planets, $\frac{d N}{d M} \propto M^{-1.16}$ (Butler et al. 2006), we can constrain what types of power laws for semi-major axis are allowed by our survey null result. To accomplish this, we simulate planets using a grid of power law indices and upper cut-offs for semi-major axis for each of our target stars. Then, the sum of the detection fractions over the entire survey gives the expected number of detected planets, assuming each star has one planet (for example, if for 10 stars, we had a 50\% chance of detecting a planet around each star, we'd expect to detect 5 planets after observing all 10 stars). Since we've set the distribution of planets, we can determine the actual planet fraction: radial velocity surveys tell us this value is $5.5 \%$ for planets more massive than 1.6 $M_{J u p}$ and with periods shorter than 4 years (closer-in than $2.5 \mathrm{AU})$ (Fischer \& Valenti 2005). We can then use the mass and semi-major axis power laws to find the planet fraction for planets down to 0.5 Jupiter masses and out to the given semi-major axis cut-off, while always preserving the value of $5.5 \%$ for the planet fraction for planets $>1.6 \mathrm{M}_{J u p}$ and $<2.5 \mathrm{AU}$. Then, by multiplying this planet fraction by the sum of detection probabilities, we find the expected number of planets we'd detect given each distribution. At this point, we can again use the Poisson distribution to convert this to a confidence level (CL) for rejecting the model, given our null result: $C L=1-e^{-\mu}$, where $\mu$ is the expected number of planets for that model. 
Since stellar multiplicity is likely to disrupt planet formation, we exclude all known stellar binaries from our target list with projected separations less than 200 AU. Since our results deal mainly with the inner 100 AU around our target stars, binaries that are any closer would greatly influence the formation of planets at these radii, creating an entirely different population. Applying this additional condition to our sample, we remove 1 star from the Masciadri et al. (2005) survey, and 9 from the Biller et al. (2007) sample, leaving 49 stars in our sample. We give further details on the binaries in our sample in Table 3

In Fig. 14 and 15, we plot the confidence with which we can reject the model for various combinations of power law index and upper cut-off for the semi-major axis distribution. For the favored model of a power law distribution given by $\frac{d N}{d a} \propto a^{-0.61}$, we can place, at the $95 \%$ confidence level, an upper-limit on the semi-major axis cut-off of 70 AU (110 AU using the models of Baraffe et al. (2003) instead of those of Burrows et al. (2003)). In other words, if the power law index has a value of -0.61 , there can be no planets in orbits beyond $\mathrm{a}=70$ AU at the $95 \%$ confidence level (27 AU at the $68 \%$ confidence level). In Fig. 16, we show how these assumptions of power law index compare with the distributions of known radial velocity planets, as well as to what confidence we can exclude various models.

\subsection{Testing Core Accretion Models}

We also consider more sophisticated models of planet populations, namely the core accretion models of Ida \& Lin (2004). Using their Fig. 12, we extract all the non-hot-Jupiter giant planets, and of the 200-300 resulting planets, we run our Monte Carlo simulation by, for each simulated planet, randomly selecting one planet from this figure, adopting its values of mass and semi-major axis, then assigning it the other orbital elements as usual. We consider each of the three cases modeled by Ida \& Lin (2004).

In Fig. 17 we plot the predicted number of planets detected from these three distributions. Again, the planet fraction for each curve is set to match the planet fraction of Fischer \& Valenti (2005) for planets above $1.6 M_{\text {Jup }}$ and within 2.5 AU. Since the predicted total number of planets detected range between about 0.6 and 0.9 at the end of our survey, we cannot place any strong constraints on these models from our null result. For the three cases of Ida \& Lin (2004), A, B, and C, we can only rule them out at the confidence levels of $48 \%, 57 \%$, and $53 \%$ respectively, and again only after leaving all binaries in the sample. Additionally, since we are considering target stars of all spectral type, we are not staying faithful to the original simulations of Ida \& Lin (2004), which consider only solar mass host stars. 


\section{Discussion: Systematic Effects of Models on Results, and Other Work}

We underscore the dependence of these results upon the accuracy of the mass-luminosity relations of Burrows et al. (2003) and Baraffe et al. (2003). In particular, these models utilize the "Hot Start" method for giant planet formation, at odds with the core accretion mechanism suggested by the planet-metallicity relation of Fischer \& Valenti (2005). The giant planet models of Marley et al. (2007) incorporate formation by core accretion, and predict systematically fainter fluxes for these young planets (typically $\sim 3$ times fainter for a $30 \mathrm{Myr}, 4 \mathrm{M}_{\text {Jup }}$ planet, so the overall effect is difficult to predict without detailed models and spectra). Another result of moving to these models, however, would be that these planets are also cooler, so that the SDI method (limited to objects with effective temperatures lower than $1400 \mathrm{~K}$ ) will likely reach planets of higher masses than would be predicted by the models of Burrows et al. (2003) and Baraffe et al. (2003).

It is possible to envision a scenario with extrasolar planets being built by both disk instability (e.g. Boss (2007)) and core accretion, with the two types of planets segregated in orbital distance: inner planets being more common in orbit around metal-rich stars, consistent with core accretion, while outer planets (the type to which the surveys discussed here are sensitive) form by disk instability. In that case, the use of the Hot Start models would be entirely reasonable, as these models have been shown to be mostly consistent with young,

low-mass objects that likely form in this way (e.g. Stassun et al. (2007), Close et al. (2007a)). This possibility (which we again note is pure speculation) endangers any conclusions drawn from Fig. 14 and 15, which assume a single, consistent population of planets, not allowing for the possibility of two overlapping populations (such as one described by broken power laws). Our results for the upper limit on planet fraction would remain valid, however, since these make no assumptions on extrasolar planet populations beyond the eccentricity distribution (a minor factor) and the mass-luminosity relation.

Clearly, these constraints would be stronger with a larger sample size to improve our statistics. Such an increase in sample size is hampered by the limited number of young, nearby stars: observing older targets tends to require an order of magnitude increase in number of targets so as to assure a similar number of detected planets. The greatest improvement in these results is likely to come with more advanced planet-finding techniques, which increase the contrast and inner working angle to which one can detect planets close to their parent stars. Two such systems, slated to come online in the next several years, are VLT-SPHERE and the Gemini Planet Imager (GPI). Surveys of a sample of young, nearby stars (likely very similar to the target list of this work) with these planet-finders should be able to greatly close the gap between the sensitivities to planets of direct imaging and radial velocity surveys. A survey planned for the immediate future is the NICI (Near 
Infrared Coronographic Imager) instrument currently being commissioned on the Gemini South Telescope, with plans for a 50-night survey for extrasolar giant planets. It is hoped, of course, that these future surveys will produce actual detections, not just more null results, which when considered alongside the targets that were not found to harbor planets, should continue to constrain parameter space on the distribution of outer extrasolar giant planets.

Another direct imaging survey for giant planets has recently been completed, searching for companions to 79 young, nearby stars: the Gemini Deep Planet Survey (Lafreniere et al. 2007). For completeness, we run an extra set of simulations to compare our results to theirs. Lafreniere et al. (2007) consider the case of planets with masses between 0.5 and $13 \mathrm{M}_{\text {Jup }}$, governed by a power law of index -1.2 (quite similar to our value of -1.16), and with a power law of index -1 for semi-major axis. They then set an upper limit on the planet fraction in three ranges of semi-major axis: $29 \%$ for $10-25$ AU, $13 \%$ for $25-50$ AU, and $9 \%$ for $50-200$ AU, all at the $95 \%$ confidence level, using the models of Baraffe et al. (2003). Adopting these same simulation parameters, we find upper limits on planet fractions of $43 \%, 29 \%$, and $34 \%$, respectively. We attribute our somewhat lower sensitivity to the increased number of stars in the Lafreniere et al. (2007) survey, as well as their increased field of view (9" compared to the 2.2" for SDI), which makes their method better-suited to detecting planets at the very large orbital radii of the last two bins. Also, the Lafreniere et al. (2007) survey was more consciously focused on closer stars: all of their target stars are within 35 parsecs, 18 of our 59 stars are beyond $35 \mathrm{pc}$. The overall results of our surveys, however, are in good agreement for the case of planets in shorter orbits: for example, we reach the same upper limits as Lafreniere et al. (2007) reached at the 95\% confidence level, if we degrade our confidence level to $87 \%$ for 10-25 AU, 75\% for 25-50 AU, and 55\% for 50-200 AU. Hence the conclusions from both surveys are the same: giant planets are rare at large separations.

We also note that the value of the planet fraction in these intervals can be estimated from the uniform detectability sample of Fischer \& Valenti (2005), which gives 5.5\% of stars having planets within 2.5 AU, and more massive than $1.6 \mathrm{M}_{J u p}$. When using a model of planet mass with index -1.2 , and semi-major axis power law index -1 , as above, the planet fractions for the semi-major axis bins 10-25 AU, 25-50 AU, and 50-200 AU become 2.1\%, $1.6 \%$, and $3.2 \%$, respectively. It should be noted that the samples of Fischer \& Valenti (2005) and Lafreniere et al. (2007) (as well as the one discussed in this paper, for that matter) are not directly comparable, as the Fischer \& Valenti (2005) sample is made up primarily of older stars (>1 Gyr), and exclusively FGK spectral types, whereas the sample of Lafreniere et al. (2007) is made up of younger stars, and contains stars of M spectral type. These two effects push the planet fractions in opposite directions: younger stars are more likely to be metal- 
rich1, and so have a higher planet fraction (Fischer \& Valenti 2005), whereas M stars are less likely to harbor giant planets (Johnson et al. 2007). Overall, then, the upper limits from both direct imaging surveys are consistent with the predictions from radial velocity detections, with respect to this particular model of planet populations.

Finally, we note that although four of our target stars do, in fact, harbor extrasolar planets (HIP 30034 (AB Pic) has a wide (5.5") companion at the planet/brown dwarf boundary, while Eps Eri, HD 81040, and HD 128311 all have radial velocity planets), our survey can be regarded as a null result. Even though these planets were orbiting our target stars, we were unable to detect them, as they were either outside our field of view (as with AB Pic B), or too faint (due to their host star's age) to be detected from our images, as was the case with the radial velocity planets. The motivation behind our simulations is to find what population of hidden (undetected) planets are consistent with a lack of planet detections, and the knowledge of existing planets around some target stars does not change this.

\section{Conclusion}

Even without detecting extrasolar planets from our surveys, the null results provide a basis for setting limits on the allowable distribution of giant planets. From our data, using the planet models of Burrows et al. (2003), we can exclude any model for planet distributions where more than $20 \%$ of stars of all spectral types have planets more massive than $4 M_{J u p}$ between 15 and $100 \mathrm{AU}$, at 95\% confidence (this upper limit becomes 8\% of stars with such planets at the $68 \%$ confidence level). If we create simple models of planet populations with the semi-major axis distribution governed by the power law $\frac{d N}{d a} \propto a^{\alpha}$, and mass by $\frac{d N}{d M} \propto M^{-1.16}$, we can exclude giant planets in the case of $\alpha=0$ beyond $16 \mathrm{AU}$, and with $\alpha=-0.5$ beyond 42 $A U$. Using the distribution of Cumming et al. (200\%), based on radial velocity observations, with $\alpha=-0.61$, there can be no giant planets beyond $70 \mathrm{AU}$. All these statements are at the 95\% confidence level; for the $68 \%$ confidence level, these upper limits for the outer cut-offs of giant planets become $11 \mathrm{AU}, 20 \mathrm{AU}$, and $27 \mathrm{AU}$, for power law indices of 0, -0.5, and -0.61 , respectively. With our data, the most we can say of the models of Ida \& Lin (2004) is that they are consistent with our observations at the $\sim 50 \%$ confidence level.

\footnotetext{
${ }^{1}$ Although Table 1 of Lafreniere et al. (2007) gives the metallicity for most of their target stars, which give a median value of $[\mathrm{Fe} / \mathrm{H}]=0$, more metal poor than the overall sample of Fischer \& Valenti (2005) by $\sim 0.1 \mathrm{dex}$, it is notoriously difficult to make accurate metallicity measurements of young stars. As a result, it is likely that these reported metallicities are systematically lower than their actual values.
} 
We thank Eric Mamajek for a great deal of assistance both in selecting targets for the SDI targets, and determining the ages of our target stars. We thank Remi Soummer for the idea of presenting sensitivity to planets as a grid of mass and semi-major axis points, and we thank Daniel Apai for presenting the idea of constructing a grid of semi-major axis power law indices and cut-offs. This work makes use of data from the European Southern Observatory, under Program 70.C - 0777D, 70.C - 0777E, 71.C-0029A, 74.C-0548, 74.C-0549, and 76.C-0094. This publication makes use of data products from the Two Micron All-Sky Survey, which is a joint project of the University of Massachusetts and the Infrared Processing and Analysis Center/California Institute of Technology, funded by the National Aeronautics and Space Administration and the National Science Foundation. This research has made use of the SIMBAD database, operated at CDS, Strasburg, France. ELN is supported by a Michelson Fellowship. LMC is supported by an NSF CAREER award and the NASA Origins of the Solar System program. BAB is supported by the NASA GSRP grant NNG04GN95H and NASA Origins grant NNG05GL71G.

\section{REFERENCES}

Abt, H. A. 1985, ApJS, 59, 95

Aitken, R. G. \& Doolittle, E. 1932, New general catalogue of double stars within 120 of the North pole ... ([Washington, D.C.] Carnegie institution of Washington, 1932.)

Alcala, J. M., Krautter, J., Schmitt, J. H. M. M., Covino, E., Wichmann, R., \& Mundt, R. 1995, A\&AS, 114, 109

Baize, P. 1950, Journal des Observateurs, 33, 1

Baraffe, I., Chabrier, G., Barman, T. S., Allard, F., \& Hauschildt, P. H. 2003, A\&A, 402, 701

Benedict, G. F., McArthur, B. E., Gatewood, G., Nelan, E., Cochran, W. D., Hatzes, A., Endl, M., Wittenmyer, R., Baliunas, S. L., Walker, G. A. H., Yang, S., Kürster, M., Els, S., \& Paulson, D. B. 2006, AJ, 132, 2206

Bennett, N. W. W., Evans, D. S., \& Laing, J. D. 1967, MNRAS, 137, 107

Bidelman, W. P. 1951, ApJ, 113, 304

Biller, B. A., Close, L. M., Masciadri, E., Nielsen, E. L., Lenzen, R., Brandner, W., MCCarthy, D., Hartung, M., Kellner, S., Mamajek, E. E., Henning, T., Miller, D., Kenworthy, M., \& Kulesa, C. 2007, accepted to ApJ 
Biller, B. A., Kasper, M., Close, L. M., Brandner, W., \& Kellner, S. 2006, ApJ, 641, L141

Bonfils, X., Forveille, T., Delfosse, X., Udry, S., Mayor, M., Perrier, C., Bouchy, F., Pepe, F., Queloz, D., \& Bertaux, J.-L. 2005, A\&A, 443, L15

Boss, A. P. 2007, ApJ, 661, L73

Brandeker, A., Jayawardhana, R., Ivanov, V. D., \& Kurtev, R. 2006, ApJ, 653, L61

Burrows, A., Sudarsky, D., \& Lunine, J. I. 2003, ApJ, 596, 587

Butler, R. P., Vogt, S. S., Marcy, G. W., Fischer, D. A., Wright, J. T., Henry, G. W., Laughlin, G., \& Lissauer, J. J. 2004, ApJ, 617, 580

Butler, R. P., Wright, J. T., Marcy, G. W., Fischer, D. A., Vogt, S. S., Tinney, C. G., Jones, H. R. A., Carter, B. D., Johnson, J. A., McCarthy, C., \& Penny, A. J. 2006, ApJ, 646,505

Chauvin, G., Lagrange, A.-M., Dumas, C., Zuckerman, B., Mouillet, D., Song, I., Beuzit, J.-L., \& Lowrance, P. 2004, A\&A, 425, L29

Chauvin, G., Lagrange, A.-M., Zuckerman, B., Dumas, C., Mouillet, D., Song, I., Beuzit, J.-L., Lowrance, P., \& Bessell, M. S. 2005, A\&A, 438, L29

Christian, D. J. \& Mathioudakis, M. 2002, AJ, 123, 2796

Close, L. M., Lenzen, R., Guirado, J. C., Nielsen, E. L., Mamajek, E. E., Brandner, W., Hartung, M., Lidman, C., \& Biller, B. 2005, Nature, 433, 286

Close, L. M., Thatte, N., Nielsen, E. L., Abuter, R., Clarke, F., \& Tecza, M. 2007a, ArXiv Astrophysics e-prints, astro-ph/0703564

Close, L. M., Zuckerman, B., Song, I., Barman, T., Marois, C., Rice, E. L., Siegler, N., Macintosh, B., Becklin, E. E., Campbell, R., Lyke, J. E., Conrad, A., \& Le Mignant, D. 2007b, ApJ, 660, 1492

Cowley, A. P., Hiltner, W. A., \& Witt, A. N. 1967, AJ, 72, 1334

Cumming et al. 2007, in prep

Cutispoto, G., Pallavicini, R., Kuerster, M., \& Rodono, M. 1995, A\&A, 297, 764 
Cutri, R. M., Skrutskie, M. F., van Dyk, S., Beichman, C. A., Carpenter, J. M., Chester, T., Cambresy, L., Evans, T., Fowler, J., Gizis, J., Howard, E., Huchra, J., Jarrett, T., Kopan, E. L., Kirkpatrick, J. D., Light, R. M., Marsh, K. A., McCallon, H., Schneider, S., Stiening, R., Sykes, M., Weinberg, M., Wheaton, W. A., Wheelock, S., \& Zacarias, N. 2003, 2MASS All Sky Catalog of point sources. (The IRSA 2MASS All-Sky Point Source Catalog, NASA/IPAC Infrared Science Archive. http://irsa.ipac.caltech.edu/applications/Gator/)

Eggen, O. J. 1996, VizieR Online Data Catalog, 5008, 0

Evans, D. S. 1961, Royal Greenwich Observatory Bulletin, 48, 389

Fabricius, C. \& Makarov, V. V. 2000, A\&A, 356, 141

Favata, F., Barbera, M., Micela, G., \& Sciortino, S. 1995, A\&A, 295, 147

Favata, F., Micela, G., \& Sciortino, S. 1997, A\&A, 322, 131

Fischer, D. A. \& Valenti, J. 2005, ApJ, 622, 1102

Gaidos, E. J., Henry, G. W., \& Henry, S. M. 2000, AJ, 120, 1006

Gliese, W. \& Jahreiß, H. 1979, A\&AS, 38, 423

Gliese, W. \& Jahreiss, H. 1991, NASA STI/Recon Technical Report A, 92, 33932

Gray, R. O., Corbally, C. J., Garrison, R. F., McFadden, M. T., Bubar, E. J., McGahee, C. E., O’Donoghue, A. A., \& Knox, E. R. 2006, AJ, 132, 161

Gray, R. O., Corbally, C. J., Garrison, R. F., McFadden, M. T., \& Robinson, P. E. 2003, AJ, 126, 2048

Henry, T. J., Soderblom, D. R., Donahue, R. A., \& Baliunas, S. L. 1996, AJ, 111, 439

Houk, N. 1978, Michigan catalogue of two-dimensional spectral types for the HD stars (Ann Arbor : Dept. of Astronomy, University of Michigan : distributed by University Microfilms International, 1978-)

Houk, N. 1982, in Michigan Spectral Survey, Ann Arbor, Dep. Astron., Univ. Michigan, 3, (1982)

Houk, N. \& Cowley, A. P. 1975, Michigan Catalogue of two-dimensional spectral types for the HD star (Ann Arbor: University of Michigan, Departement of Astronomy, 1975) 
Houk, N. \& Smith-Moore, M. 1988, in Michigan Spectral Survey, Ann Arbor, Dept. of Astronomy, Univ. Michigan (Vol. 4), (1988)

Ida, S. \& Lin, D. N. C. 2004, ApJ, 604, 388

Itoh, Y., Hayashi, M., Tamura, M., Tsuji, T., Oasa, Y., Fukagawa, M., Hayashi, S. S., Naoi, T., Ishii, M., Mayama, S., Morino, J.-i., Yamashita, T., Pyo, T.-S., Nishikawa, T., Usuda, T., Murakawa, K., Suto, H., Oya, S., Takato, N., Ando, H., Miyama, S. M., Kobayashi, N., \& Kaifu, N. 2005, ApJ, 620, 984

Jayawardhana, R., Hartmann, L., Fazio, G., Fisher, R. S., Telesco, C. M., \& Piña, R. K. 1999, ApJ, 521, L129

Johnson et al. 2007, in prep

Lachaume, R., Dominik, C., Lanz, T., \& Habing, H. J. 1999, A\&A, 348, 897

Lafreniere, D., Doyon, R., Marois, C., Nadeau, D., Oppenheimer, B. R., Roche, P. F., Rigaut, F., Graham, J. R., Jayawardhana, R., Johnstone, D., Kalas, P. G., Macintosh, B., \& Racine, R. 2007, ArXiv e-prints, 705

Leaton, B. R. \& Pagel, B. E. J. 1960, MNRAS, 120, 317

Lenzen, R., Close, L., Brandner, W., Biller, B., \& Hartung, M. 2004, in Ground-based Instrumentation for Astronomy. Edited by Alan F. M. Moorwood and Iye Masanori. Proceedings of the SPIE, Volume 5492, pp. 970-977 (2004)., ed. A. F. M. Moorwood \& M. Iye, 970-977

López-Santiago, J., Montes, D., Crespo-Chacón, I., \& Fernández-Figueroa, M. J. 2006, ApJ, 643,1160

Lowrance, P. J., Becklin, E. E., Schneider, G., Kirkpatrick, J. D., Weinberger, A. J., Zuckerman, B., Dumas, C., Beuzit, J.-L., Plait, P., Malumuth, E., Heap, S., Terrile, R. J., \& Hines, D. C. 2005, AJ, 130, 1845

Luhman, K. L., Allers, K. N., Jaffe, D. T., Cushing, M. C., Williams, K. A., Slesnick, C. L., \& Vacca, W. D. 2007, ApJ, 659, 1629

Luhman, K. L., Wilson, J. C., Brandner, W., Skrutskie, M. F., Nelson, M. J., Smith, J. D., Peterson, D. E., Cushing, M. C., \& Young, E. 2006, ApJ, 649, 894

Mamajek, E. E. 2007, private communication 
Marley, M. S., Fortney, J. J., Hubickyj, O., Bodenheimer, P., \& Lissauer, J. J. 2007, ApJ, 655,541

Masciadri, E., Mundt, R., Henning, T., Alvarez, C., \& Barrado y Navascués, D. 2005, ApJ, 625,1004

Mathioudakis, M., Drake, J. J., Craig, N., Kilkenny, D., Doyle, J. G., Sirk, M. M., Dupuis, J., Fruscione, A., Christian, C. A., \& Abbott, M. J. 1995, A\&A, 302, 422

McCaughrean, M. J., Close, L. M., Scholz, R.-D., Lenzen, R., Biller, B., Brandner, W., Hartung, M., \& Lodieu, N. 2004, A\&A, 413, 1029

Metchev, S. A. \& Hillenbrand, L. A. 2004, ApJ, 617, 1330

Montes, D., López-Santiago, J., Gálvez, M. C., Fernández-Figueroa, M. J., De Castro, E., \& Cornide, M. 2001, MNRAS, 328, 45

Nesterov, V. V., Kuzmin, A. V., Ashimbaeva, N. T., Volchkov, A. A., Röser, S., \& Bastian, U. 1995, A\&AS, 110, 367

Neuhauser, R. \& Brandner, W. 1998, A\&A, 330, L29

Neuhäuser, R., Guenther, E., Mugrauer, M., Ott, T., \& Eckart, A. 2002, A\&A, 395, 877

Nielsen, E. L., Close, L. M., Guirado, J. C., Biller, B. A., Lenzen, R., Brandner, W., Hartung, M., \& Lidman, C. 2005, Astronomische Nachrichten, 326, 1033

Noyes, R. W., Weiss, N. O., \& Vaughan, A. H. 1984, ApJ, 287, 769

Perryman, M. A. C., Lindegren, L., Kovalevsky, J., Hoeg, E., Bastian, U., Bernacca, P. L., Crézé, M., Donati, F., Grenon, M., van Leeuwen, F., van der Marel, H., Mignard, F., Murray, C. A., Le Poole, R. S., Schrijver, H., Turon, C., Arenou, F., Froeschlé, M., \& Petersen, C. S. 1997, A\&A, 323, L49

Pounds, K. A., Allan, D. J., Barber, C., Barstow, M. A., Bertram, D., Branduardi-Raymont, G., Brebner, G. E. C., Buckley, D., Bromage, G. E., Cole, R. E., Courtier, M., Cruise, A. M., Culhane, J. L., Denby, M., Donoghue, D. O., Dunford, E., Georgantopoulos, I., Goodall, C. V., Gondhalekar, P. M., Gourlay, J. A., Harris, A. W., Hassall, B. J. M., Hellier, C., Hodgkin, S., Jeffries, R. D., Kellett, B. J., Kent, B. J., Lieu, R., Lloyd, C., McGale, P., Mason, K. O., Matthews, L., Mittaz, J. P. D., Page, C. G., Pankiewicz, G. S., Pike, C. D., Ponman, T. J., Puchnarewicz, E. M., Pye, J. P., Quenby, J. J., Ricketts, M. J., Rosen, S. R., Sansom, A. E., Sembay, S., Sidher, S., Sims, M. R., Stewart, B. C., Sumner, T. J., Vallance, R. J., Watson, M. G., Warwick, R. S., Wells, 
A. A., Willingale, R., Willmore, A. P., Willoughby, G. A., \& Wonnacott, D. 1993, MNRAS, 260, 77

Poveda, A., Herrera, M. A., Allen, C., Cordero, G., \& Lavalley, C. 1994, Revista Mexicana de Astronomia y Astrofisica, 28, 43

Randich, S., Gratton, R., \& Pallavicini, R. 1993, A\&A, 273, 194

Raymond, S. N. 2006, ApJ, 643, L131

Simon, T. \& Drake, S. A. 1993, AJ, 106, 1660

Soderblom, D. R., King, J. R., \& Henry, T. J. 1998, AJ, 116, 396

Song, I., Bessell, M. S., \& Zuckerman, B. 2002, ApJ, 581, L43

Song, I., Zuckerman, B., \& Bessell, M. S. 2003, ApJ, 599, 342

Sozzetti, A., Udry, S., Zucker, S., Torres, G., Beuzit, J. L., Latham, D. W., Mayor, M., Mazeh, T., Naef, D., Perrier, C., Queloz, D., \& Sivan, J.-P. 2006, A\&A, 449, 417

Stassun, K. G., Mathieu, R. D., \& Valenti, J. A. 2007, ArXiv e-prints, 704

Torres, C. A. O., da Silva, L., Quast, G. R., de la Reza, R., \& Jilinski, E. 2000, AJ, 120, 1410

Vyssotsky, A. N., Janssen, E. M., Miller, W. J., \& Walther, M. E. 1946, ApJ, 104, 234

Waite, I. A., Carter, B. D., Marsden, S. C., \& Mengel, M. W. 2005, Publications of the Astronomical Society of Australia, 22, 29

Webb, R. A., Zuckerman, B., Platais, I., Patience, J., White, R. J., Schwartz, M. J., \& McCarthy, C. 1999, ApJ, 512, L63

Wichmann, R., Schmitt, J. H. M. M., \& Hubrig, S. 2003, A\&A, 399, 983

Wilson, Jr., R. H. 1954, AJ, 59, 132

Wright, J. T., Marcy, G. W., Butler, R. P., \& Vogt, S. S. 2004, ApJS, 152, 261

Zuckerman, B. \& Song, I. 2004, ARA\&A, 42, 685

Zuckerman, B., Song, I., Bessell, M. S., \& Webb, R. A. 2001a, ApJ, 562, L87

Zuckerman, B., Webb, R. A., Schwartz, M., \& Becklin, E. E. 2001b, ApJ, 549, L233 
This preprint was prepared with the AAS IATEX macros v5.2. 


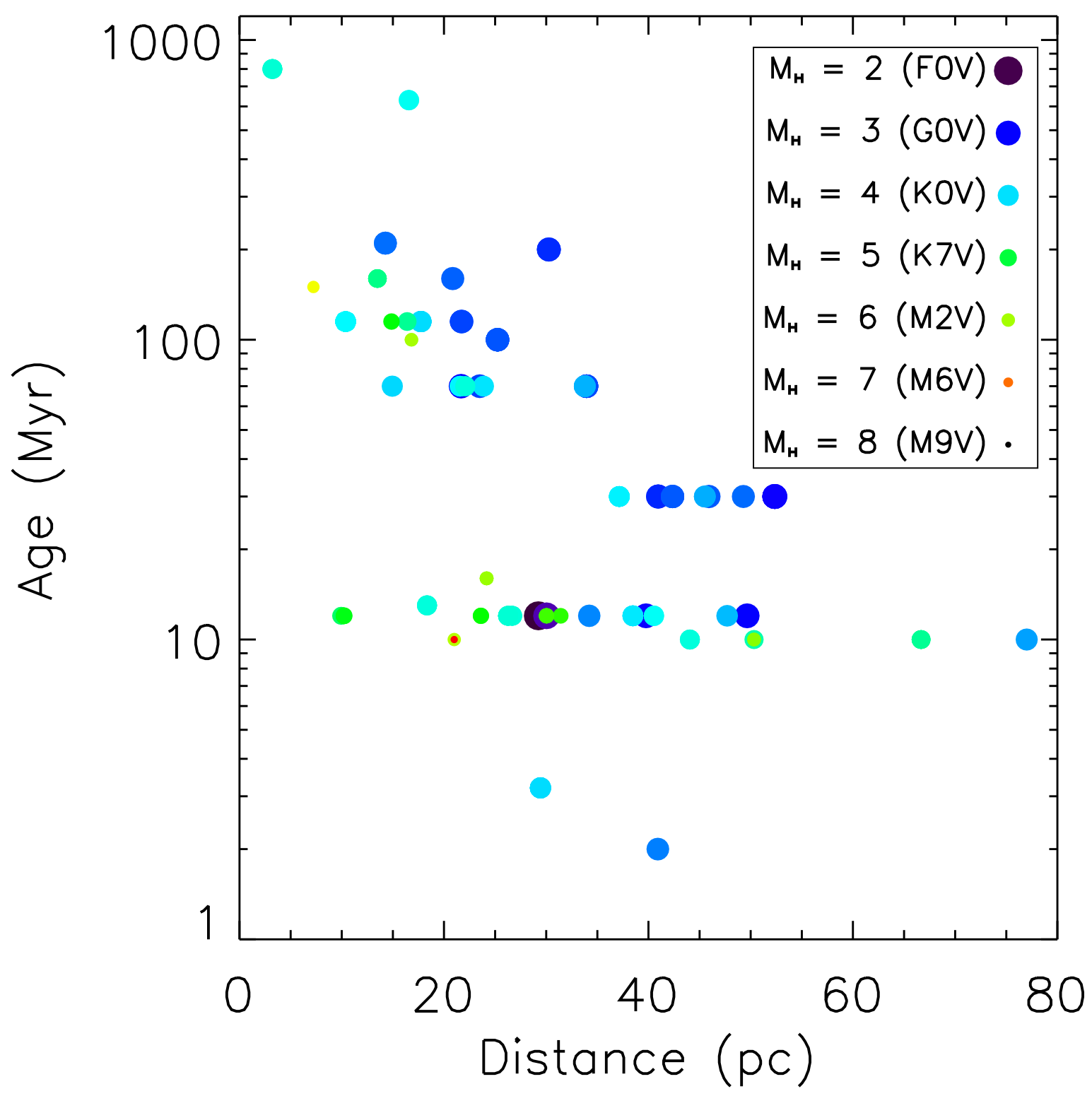

Fig. 1.- The 59 target stars from our two surveys (though five stars are too old to appear on this plot). These stars are some of the youngest, nearest stars known, spanning a range of spectral type. The size of the plotting symbol and the color is proportional to the absolute $\mathrm{H}$ magnitude of the star: a bigger, bluer symbol corresponds to a brighter and hotter star. The legend gives approximate spectral type conversions for main sequence stars, but we note that these stars have been plotted by their 2MASS H-band fluxes, and as a result their actual spectral type can vary from that shown in the legend. See Table 1 for more complete properties of these stars. The median target star is a $30 \mathrm{Myr}$ K2 star at 25 pc. 


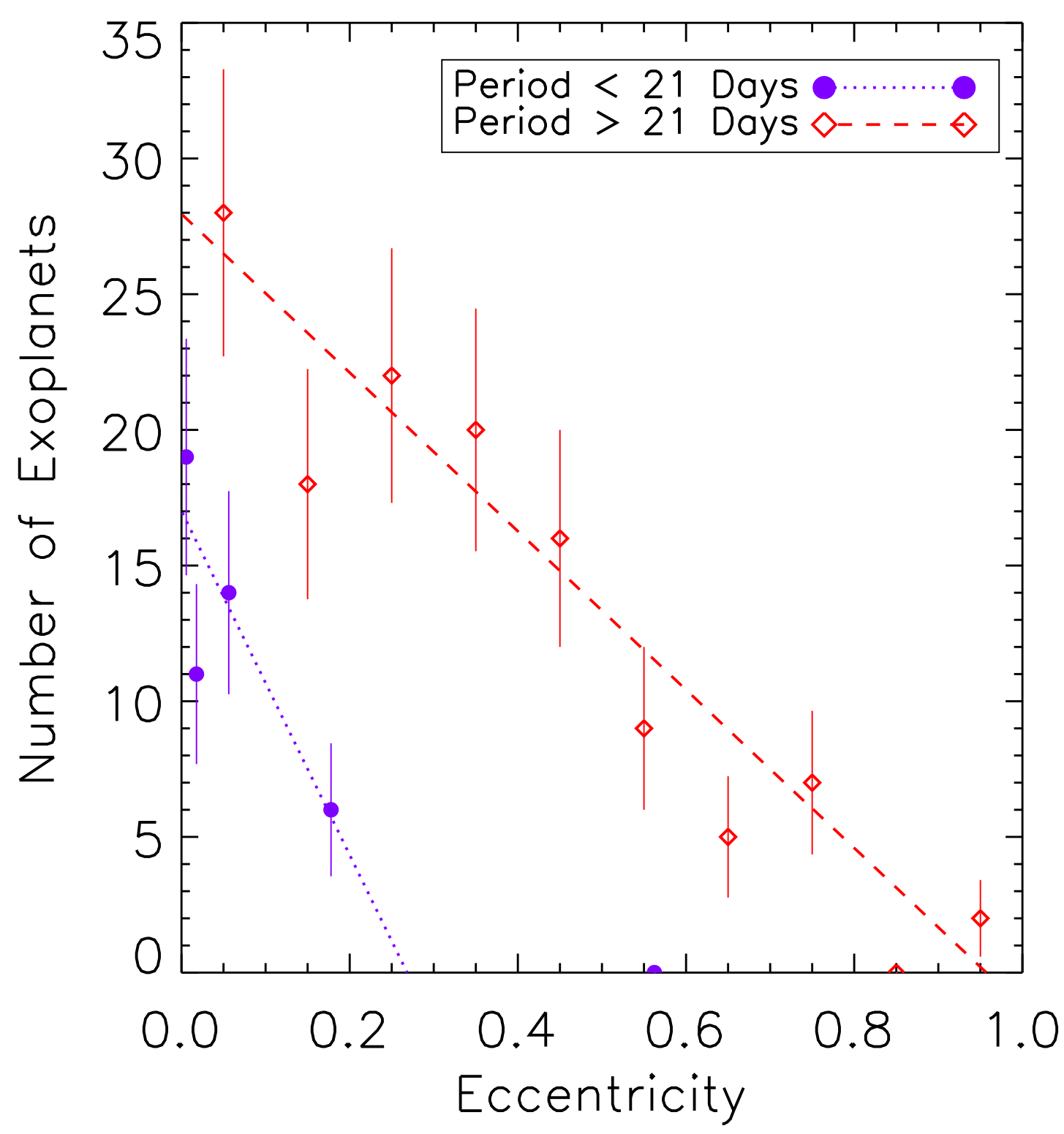

Fig. 2.- The assumed distribution for the orbital eccentricities of extrasolar planets. The datapoints represent the histograms for planets found to date with the radial velocity method (Butler et al. 2006), with error bars as 1-sigma Poisson noise based on the number of planets per bin. Planets are divided to separate "Hot Jupiters," based on a period cut at 21 days; long period planets are divided into linear bins, short-period ones into logarithmic bins. In both cases, a simple linear fit is a good representation of the data. 


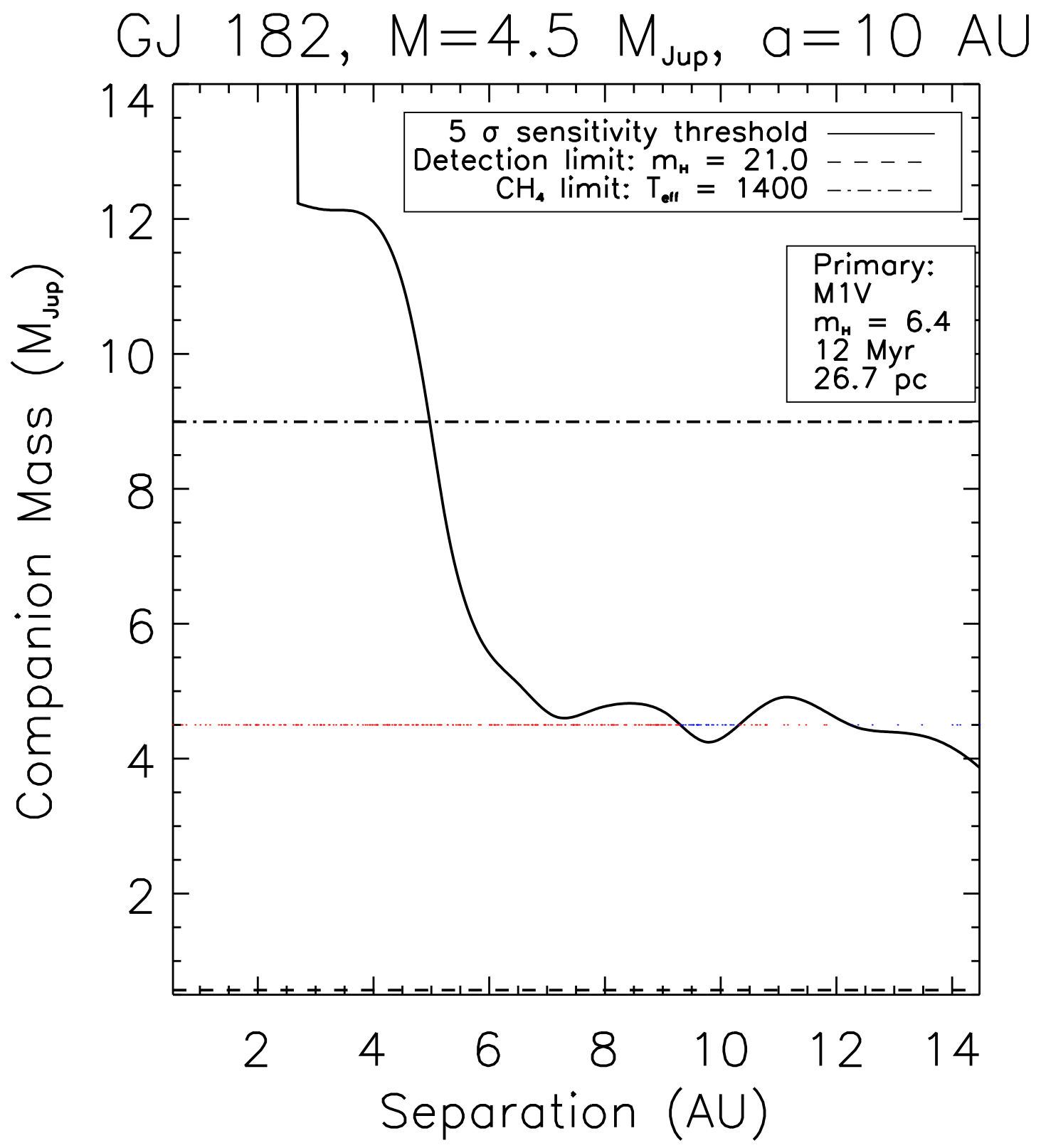

Fig. 3. - The results of a single simulation of $10^{4}$ planets around the SDI target star GJ 182 . (Biller et al. 2007) Each planet has a mass of $4.5 M_{J u p}$, and a semi-major axis of $10 \mathrm{AU}$. Due to various values of eccentricity, viewing angle, and orbital phase, the projected separation of each simulated planet departs from the semi-major axis, and the points smear across the horizontal direction, with projected separation running between 0 and $14 \mathrm{AU}$. Planets that are above the contrast curve are detected (blue dots), while those below are not (red dots). In this case, $13 \%$ of these simulated planets were detected. By running this simulation over multiple grid points of mass and semi-major axis, we produce a full completeness plot, such as Fig. 4 , 


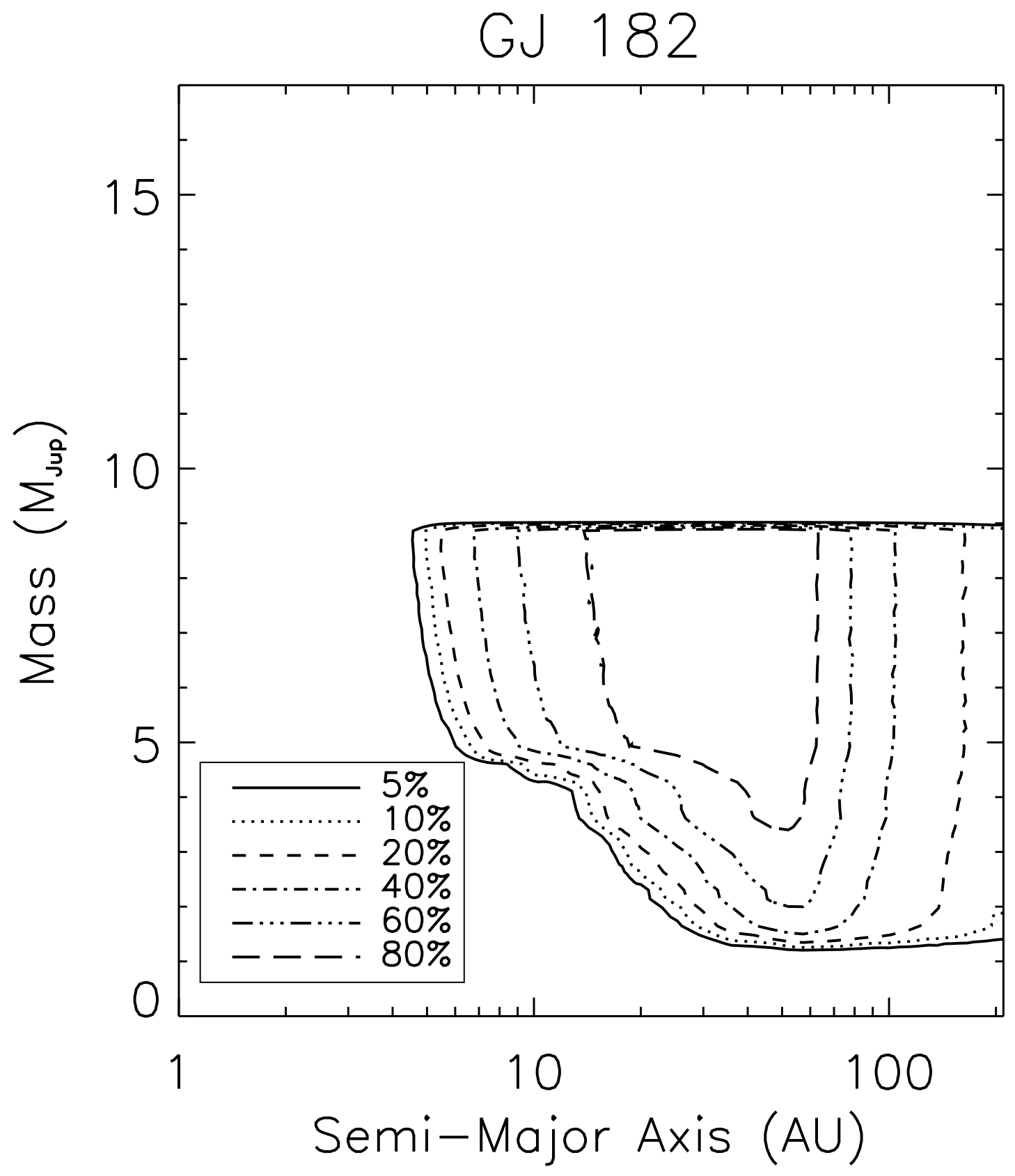

Fig. 4.- A full completeness plot for the target star GJ 182. As a function of planet mass and semi-major axis (with grid points between 0.5 and $17 \mathrm{M}_{J u p}$ for mass, and semi-major axis between 1 and $210 \mathrm{AU}$ ), the contours give the probability of detecting a planet with those parameters given the available contrast curve. At each grid point, $10^{4}$ planets are simulated, as shown in Fig. 3, and the fraction that can be detected is returned. The left edge is strongly influenced by the shape of the contrast curve, while the right edge depends mainly on the projected field of view of the observation. The hard upper limit at $9 \mathrm{M}_{J u p}$ is set by the methane cut-off imposed by the SDI method, when the simulated planets exceed $1400 \mathrm{~K}$ and cease to have a strong methane signature in the spectrum. 


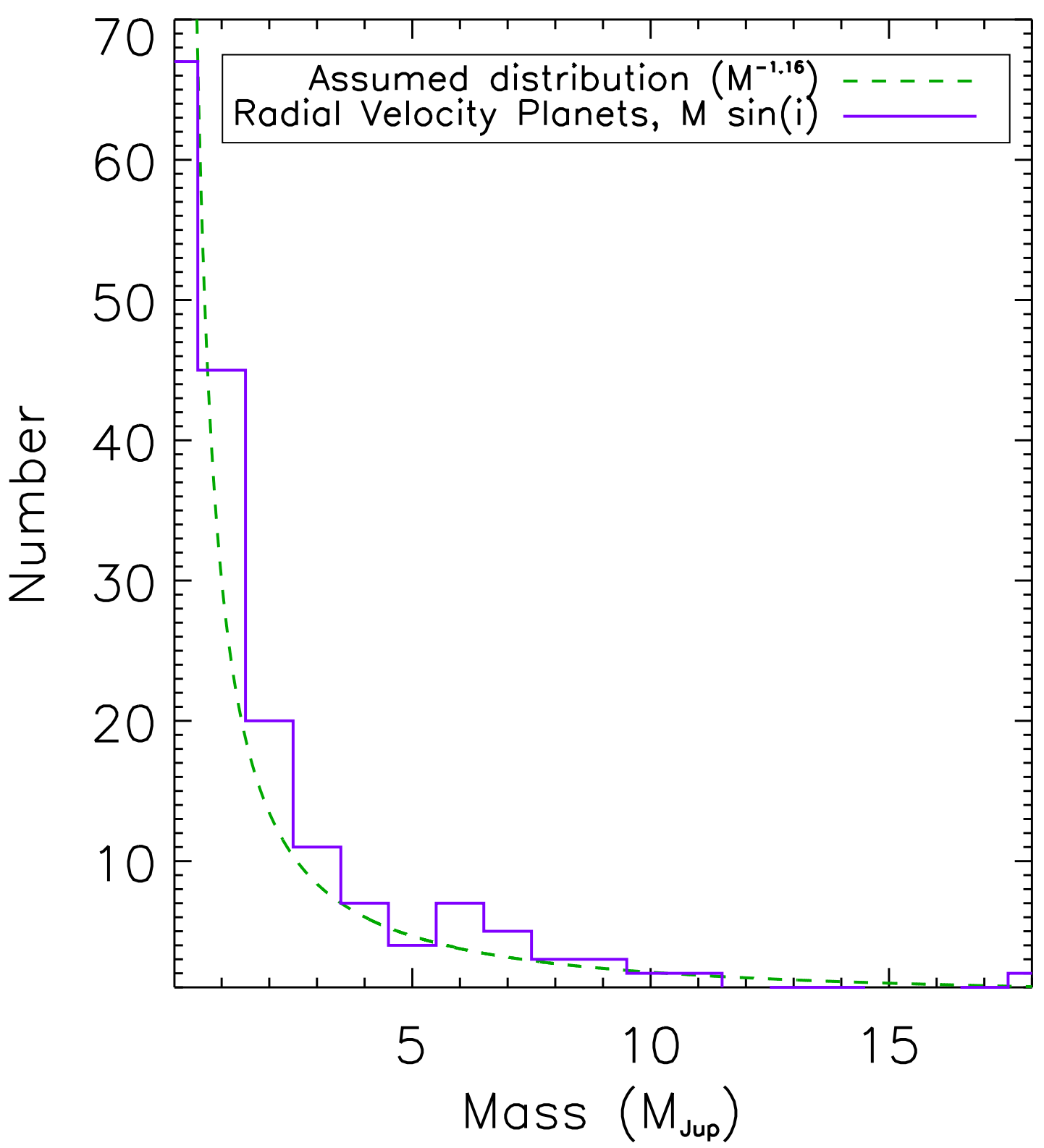

Fig. 5.- The assumed mass distribution of extrasolar planets, plotted against the histogram of known planets detected by the radial velocity method. Throughout this paper we adopt a power law of the form $\frac{d N}{d M} \propto M^{-1.16}$, as suggested by Butler et al. (2006), which does a reasonable job fitting the data. 


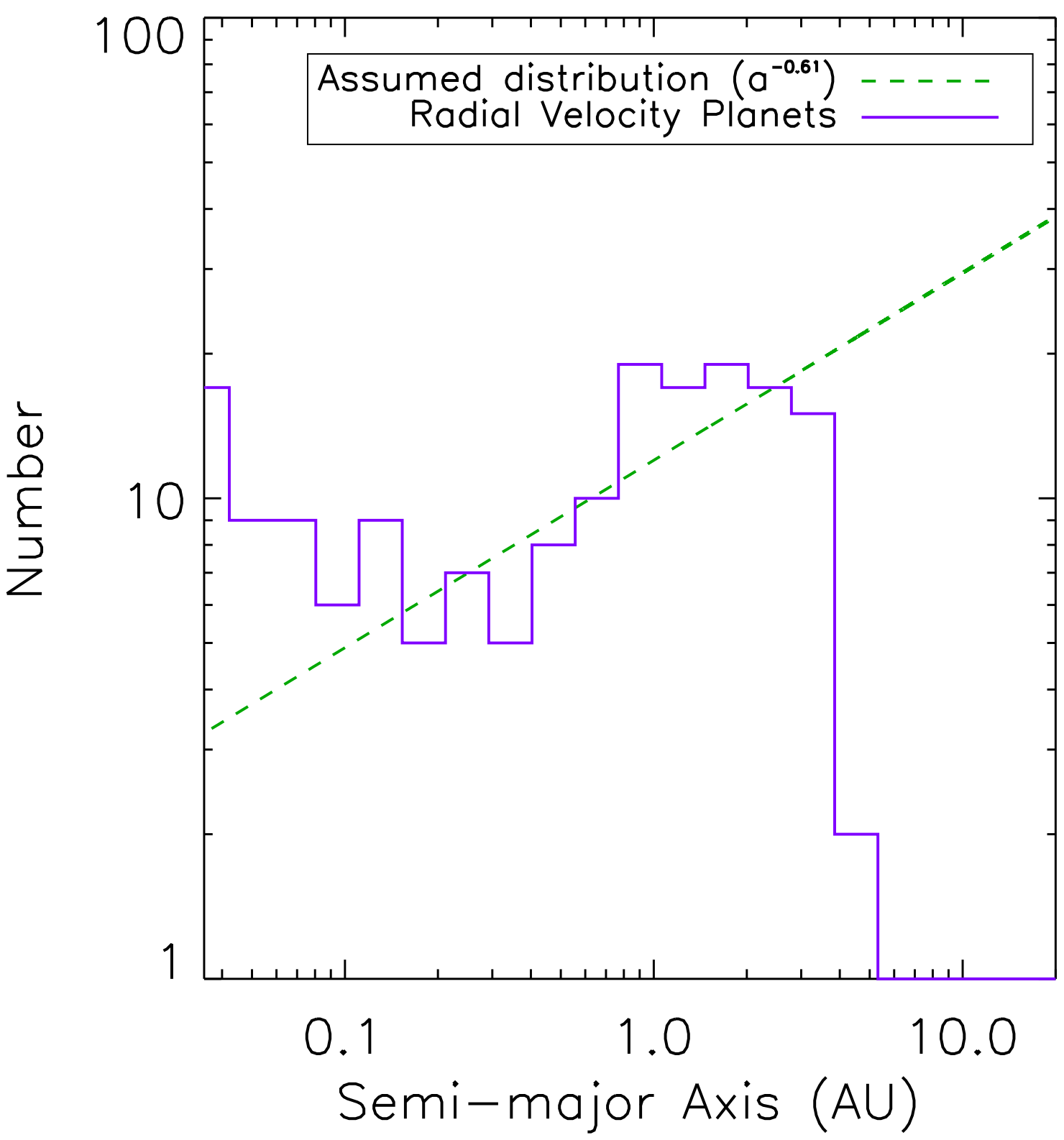

Fig. 6.- The distributions that we consider for semi-major axis of extrasolar planets, again with the histogram of known radial velocity planets. We adopt the observed distribution of Cumming et al. (2007), with $\frac{d N}{d a} \propto a^{-0.61}$, which is suggestive of the existance of wider planets, given that radial velocity surveys should be especially sensitive to hot Jupiters (producing an over-abundance at small separations) and less sensitive to long-period orbits (resulting in a decline in detected planets at larger separations). 


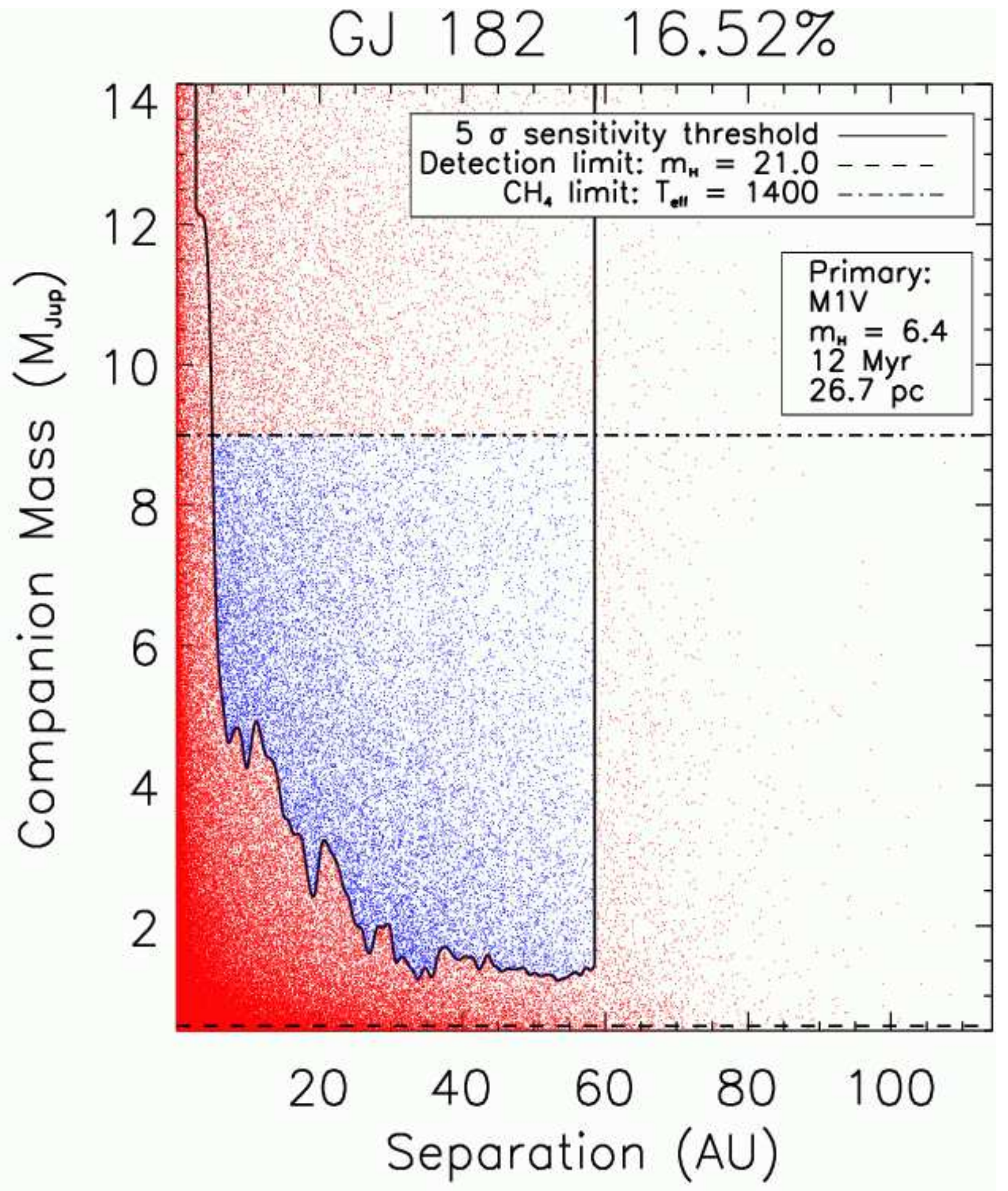

Fig. 7.- $10^{5}$ simulated planets around the SDI target star GJ 182, following the distributions for mass $\left(\frac{d N}{d !} \propto M^{-1.16}\right)$ of Butler et al. (2006) and semi-major axis $\left(\frac{d N}{d a} \propto a^{-0.61}\right)$ of Cumming et al. (2007), with mass running from 0.5 to $13 M_{J u p}$, and semi-major axis cut off at $70 \mathrm{AU}$ (since there is a range of eccentricities, separation can exceed the semi-major axis cut-off). Detected planets (blue dots) are those that lie above the contrast curve, above the minimum flux level, and below the methane cut-off. In this case, $17 \%$ of the simulated planets could be detected with this observation. Using the metric of completeness to planets 


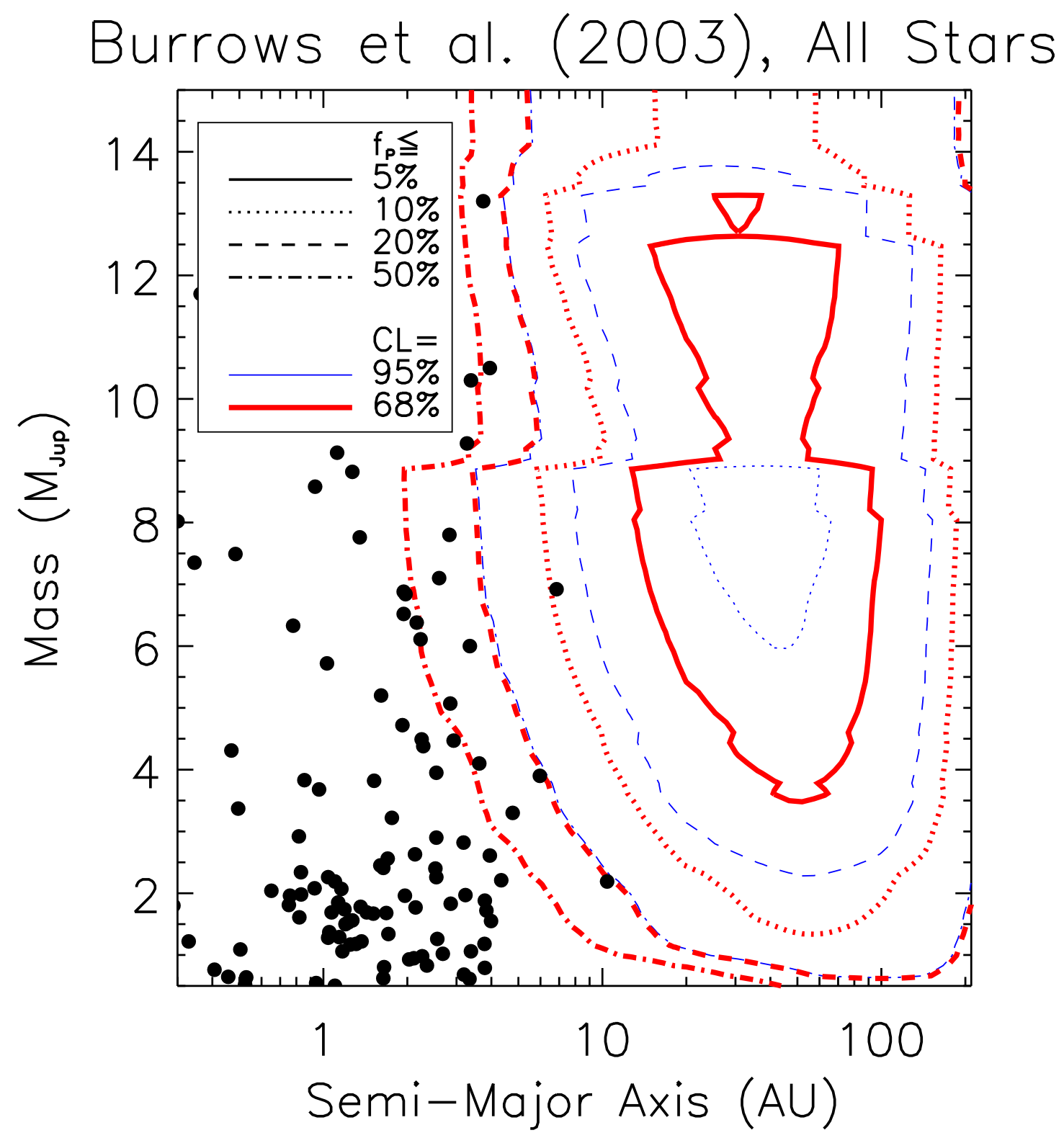

Fig. 8. - The upper limit on the fraction of stars with planets $\left(f_{p}\right)$, as a function of mass and semi-major axis (see Eq. 2), using the planet models of Burrows et al. (2003), with the $95 \%$ confidence level plotted as thin blue lines. We also plot in thicker red lines the $68 \%$ confidence level contours. Given the results of our survey, we would expect, for example, less than $20 \%$ (as indicated by the thin dashed blue line) of stars to have a planet of mass greater than $4 M_{J u p}$ in an orbit $15<\mathrm{a}<100 \mathrm{AU}$, and less than $50 \%$ of stars (the dot-dashed thin blue line) to have planets more massive than $4 M_{J u p}$ with semi-major axes between 5 and $200 \mathrm{AU}$, at the $95 \%$ confidence level. Also plotted in the solid circles are known extrasolar planets. There is still a gap between planets probed by direct imaging surveys such as the ones described in this work, and those using the radial velocity method. 


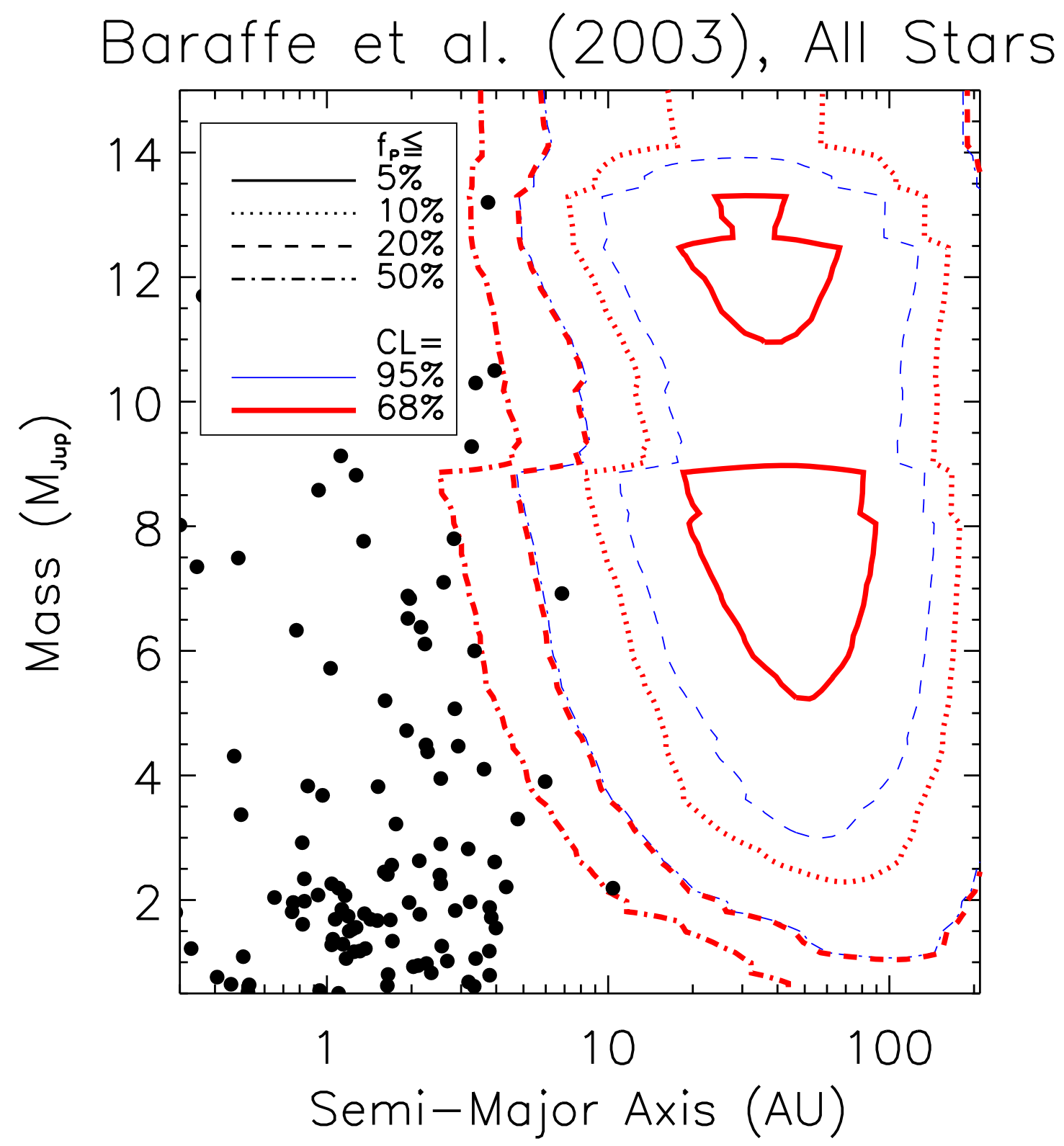

Fig. 9.- The same as Fig. 8, but instead using the models of Baraffe et al. (2003) to convert between planet mass and NIR magnitudes. The COND models generally predict higher masses for a given $\mathrm{H}$ or Ks magnitude and age. 


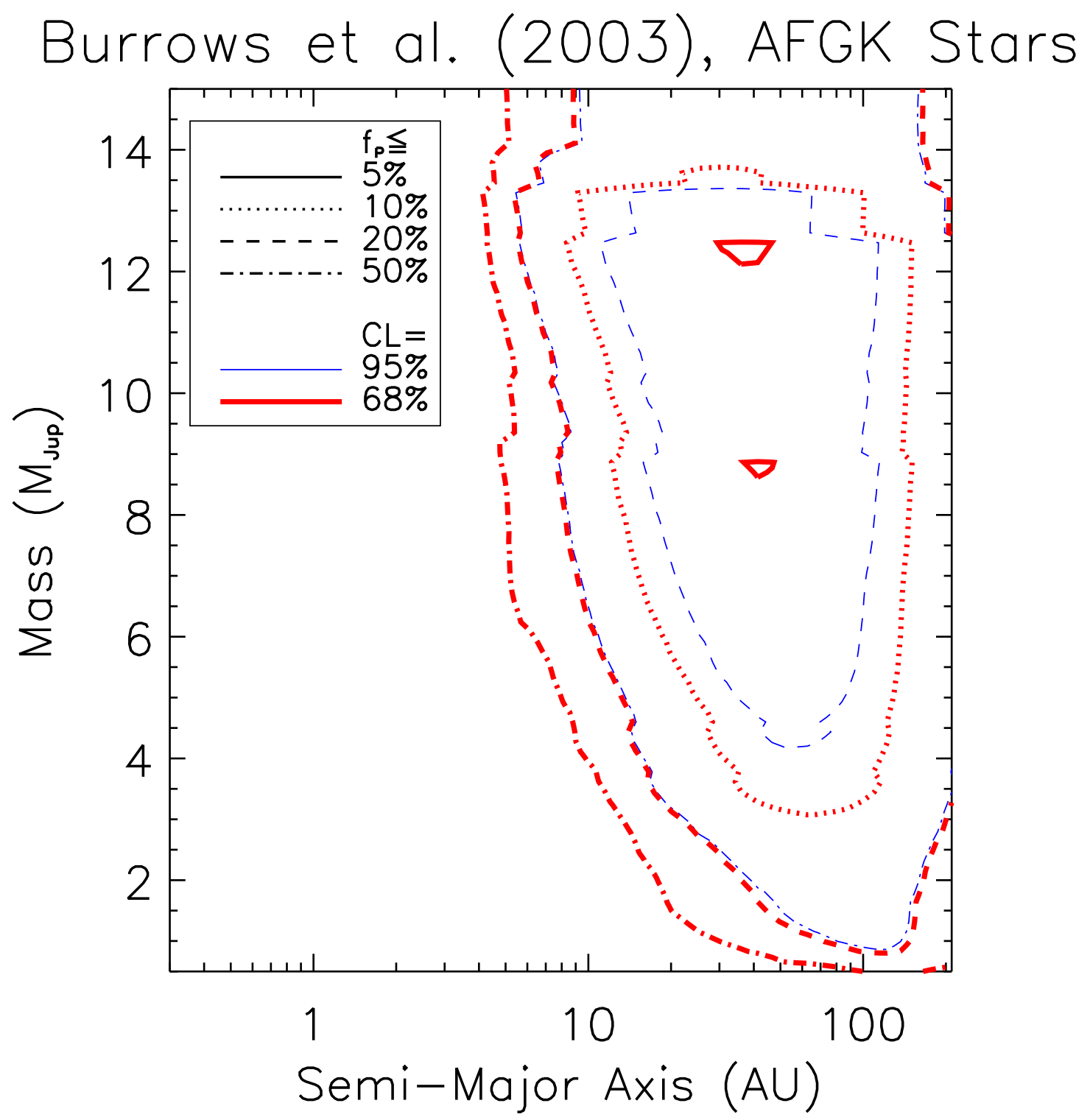

Fig. 10. - The $95 \%$ and $68 \%$ confidence upper limit on planet fraction, limited only to stars of spectral type A through K, using the Burrows et al. (2003) models. Since with earlier spectral types the parent star is intrinsically brighter, it becomes more difficult to access planets of smaller masses or smaller separations. For AFGK stars we can only say, at the $95 \%$ confidence level, that less than $20 \%$ of stars have $M>6 M_{\text {Jup }}$ planets at 30-100 AU, or a limit of $50 \%$ for planets with masses above $3 M_{J u p}$ at 20-200 AU. 


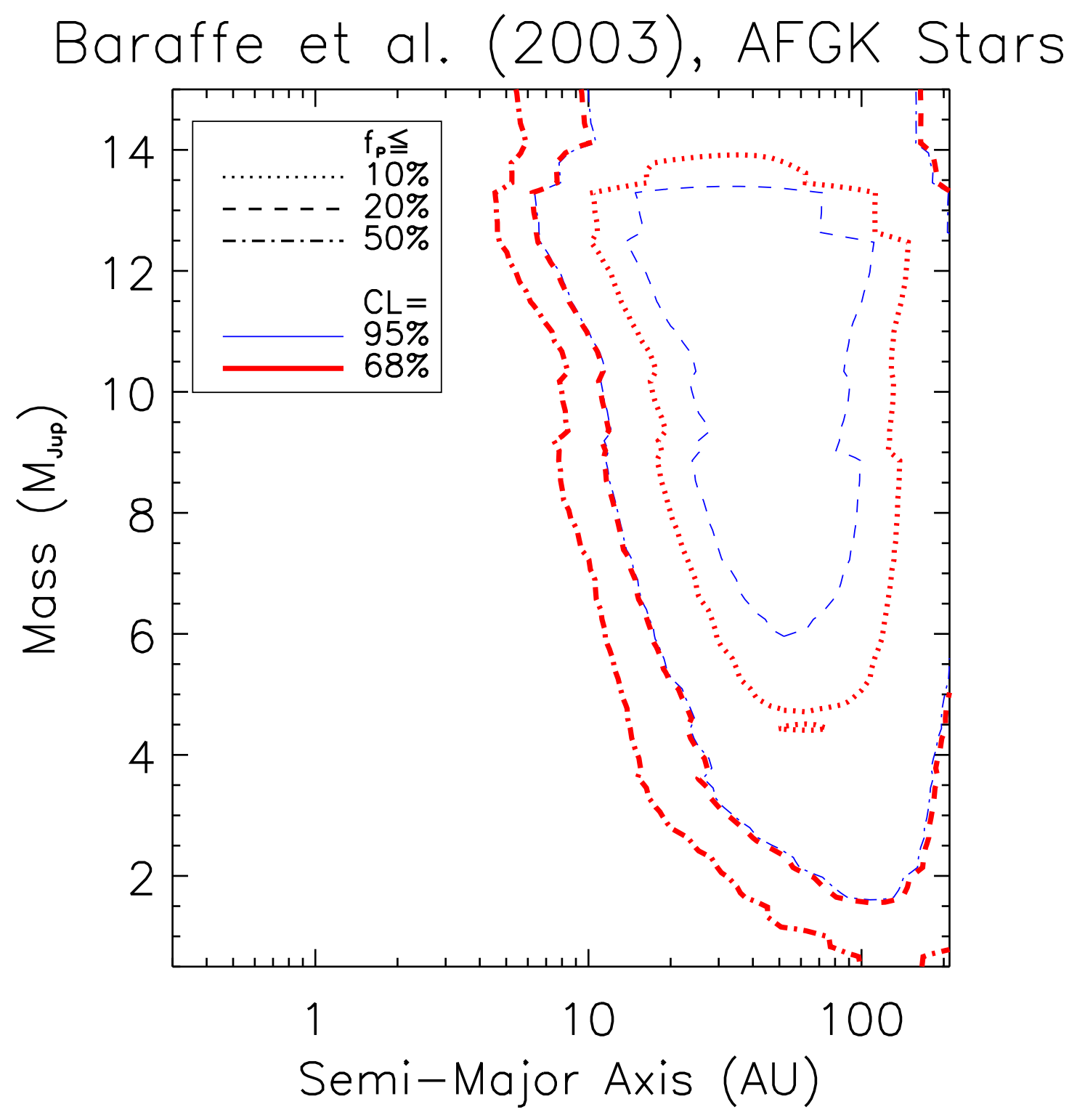

Fig. 11. - The same as Fig. 10, but with the Baraffe et al. (2003) models used to find planet masses. 


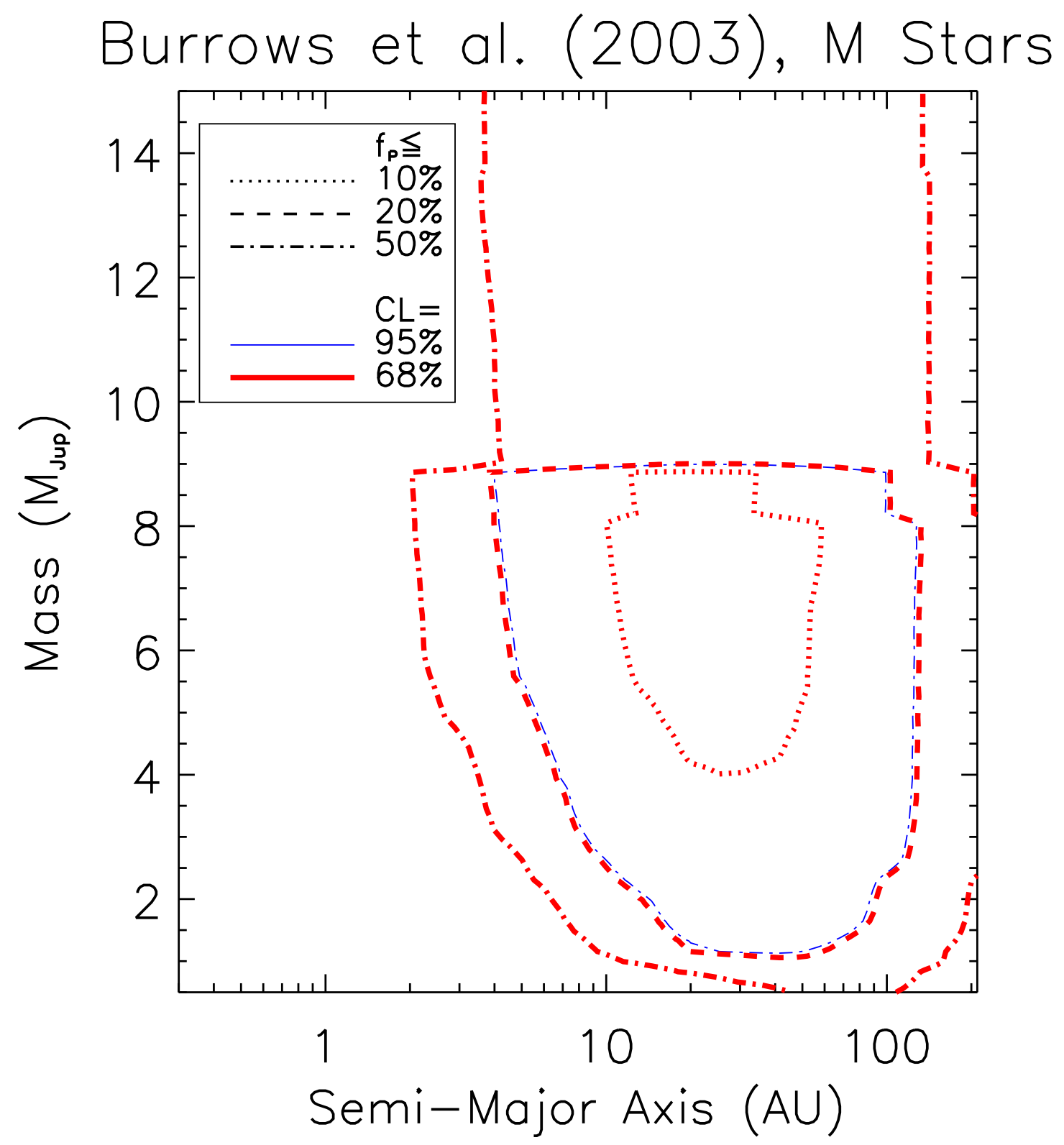

Fig. 12.- Now using only our $15 \mathrm{M}$ stars, we again plot the $95 \%$ and $68 \%$ confidence level upper limit on planet fraction, using the Burrows et al. (2003) models. While the plot follows the shape of Fig. 8, the removal of about three-quarters of the target stars reduces the upper limit that can be set on the planet fraction. Hence less than $50 \%$ of $\mathrm{M}$ stars should have planets with $2>M>4 M_{\text {Jup }}$ from 10 to $100 \mathrm{AU}$, at $95 \%$ confidence. 


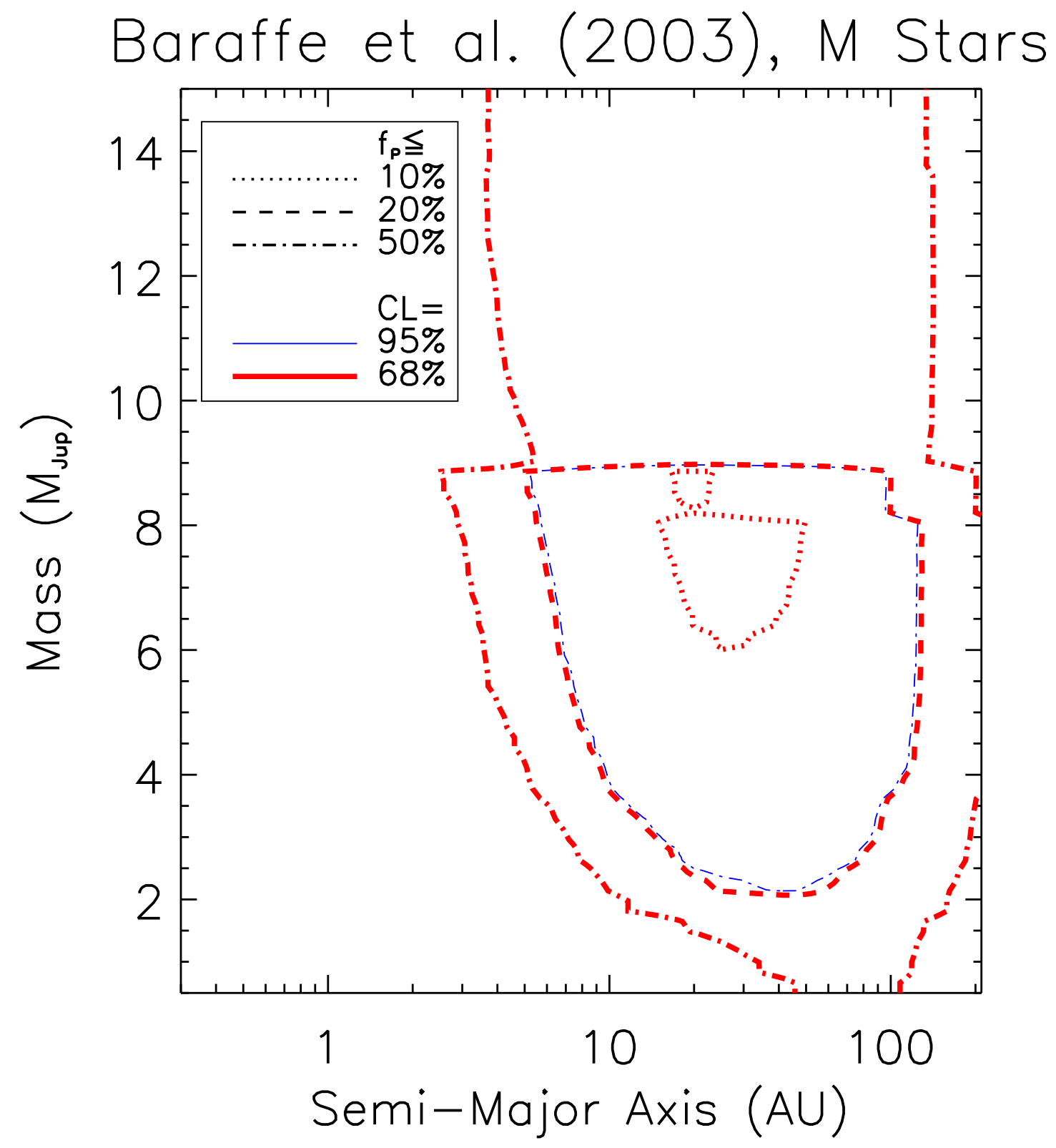

Fig. 13.- As with Fig. 12, only now with the Baraffe et al. (2003) models used to find planet masses. 


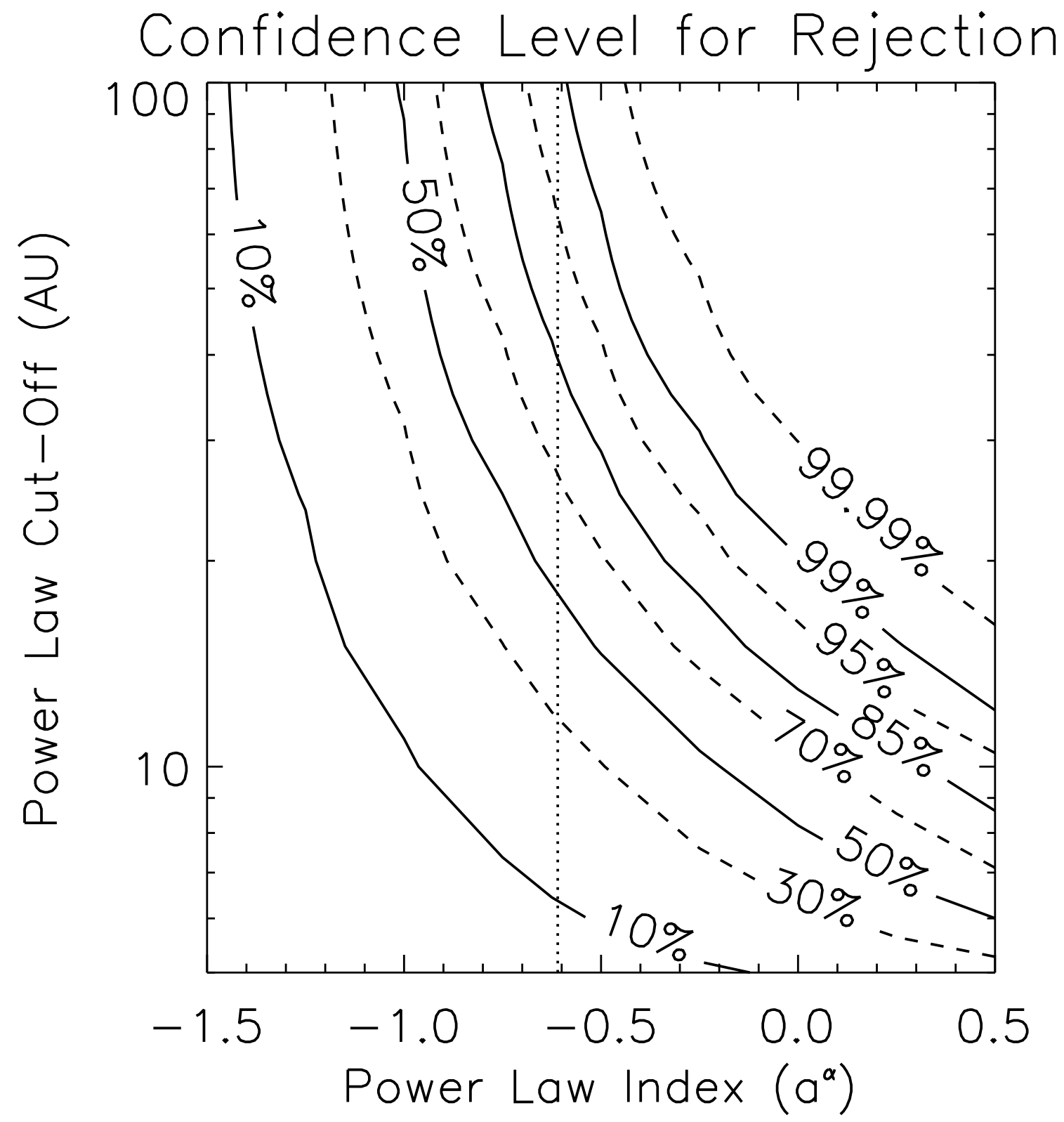

Fig. 14. - The confidence level with which we can reject models of planet populations, assuming a power-law distribution for semi-major axes $\left(\frac{d N}{d a} \propto a^{\alpha}\right)$, as a function of the power law index and upper cut-off $\left(\mathrm{N}(\mathrm{a})=0\right.$ for $\left.a \geq a_{C u t-o f f}\right)$. The expected power-law index from the radial velocity distribution (see Fig 6) is -0.61 (Cumming et al. 2007), and given these data we can place a $95 \%$ confidence limit on the upper cut-off of 70 AU. At $68 \%$ confidence, there cannot be giant planets in orbits beyond $27 \mathrm{AU}$, for this choice of power law index. For this figure, we use the models of Burrows et al. (2003) 


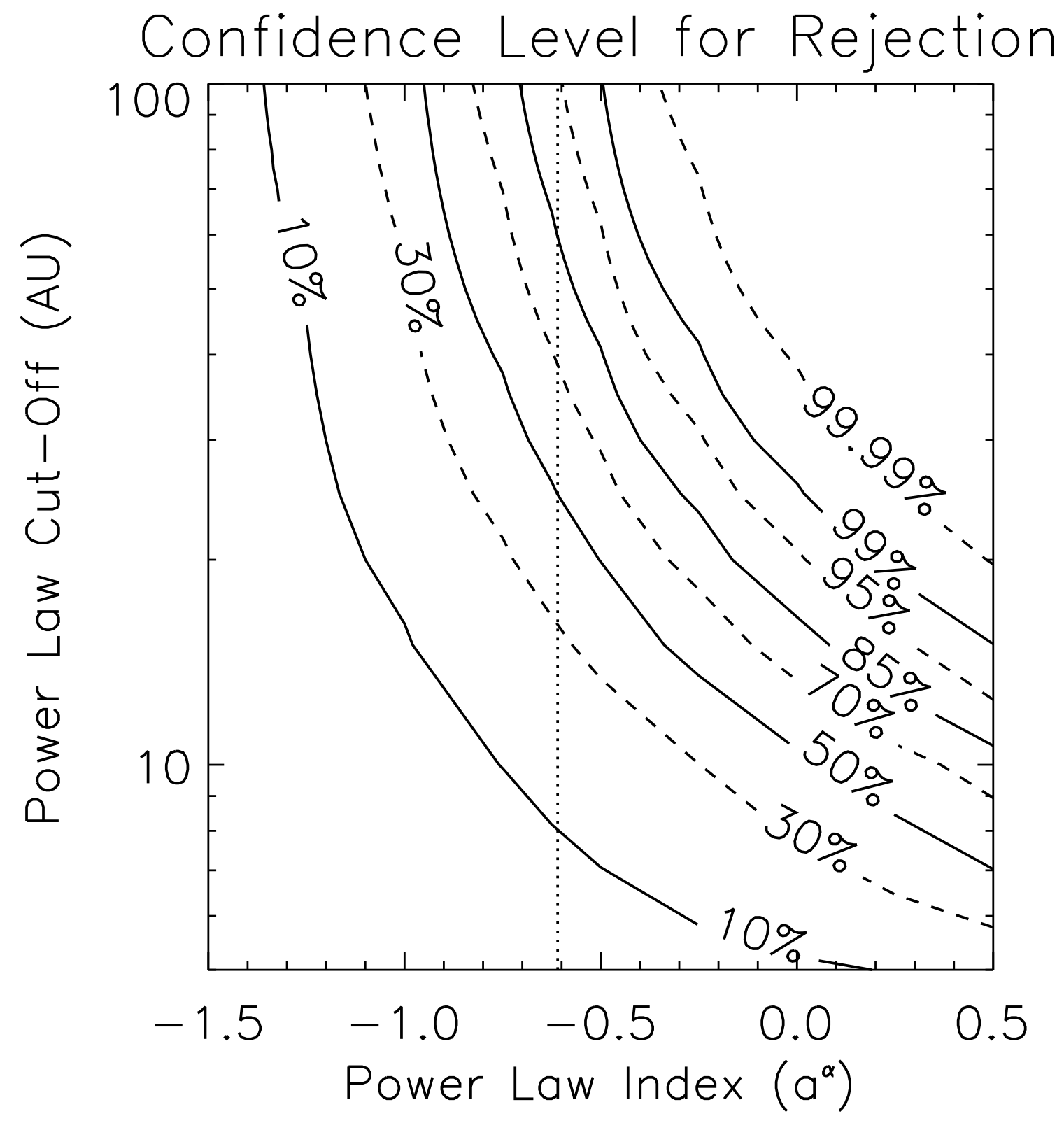

Fig. 15.- The same as Fig. 14, but using the models of Baraffe et al. (2003). The 95\% confidence upper cut-off for semi-major axis for the $\frac{d N}{d a} \propto a^{\alpha}$ model now moves to $110 \mathrm{AU}$. 


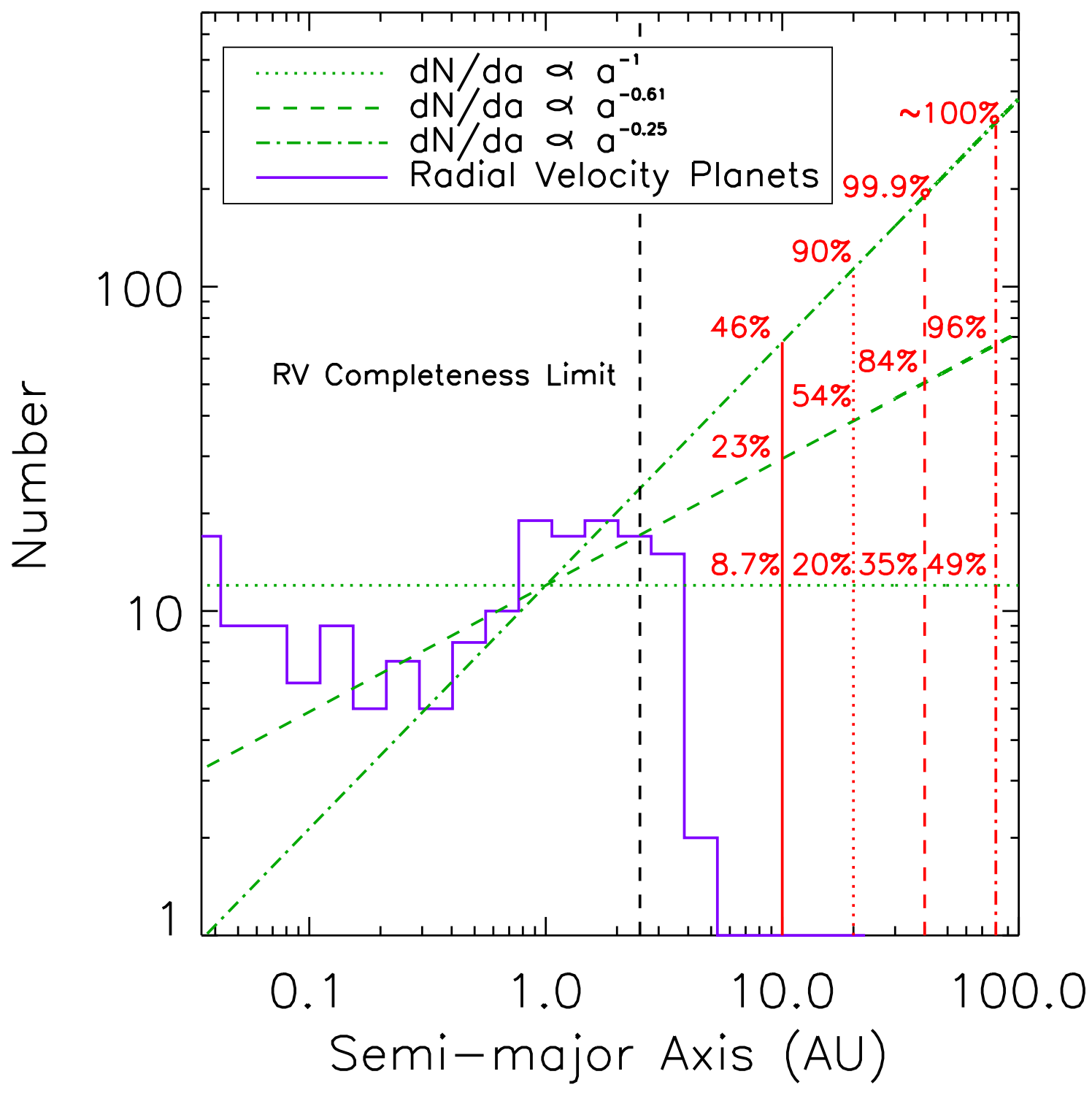

Fig. 16. - The histogram (in blue) of the distribution of known extrasolar giant planets found with the radial velocity method, plotted against a series of power laws considered in Fig. 14 and 15. Since radial velocity observations are only complete to about $2.5 \mathrm{AU}$, a less steep drop-off of planets with semi-major axis is possible. We give the confidence with which we can rule out various combinations of power law index and upper cut-off, for indices of $-1,-0.61,-0.25$, and upper cut-offs of $10 \mathrm{AU}, 20 \mathrm{AU}, 40 \mathrm{AU}$, and $80 \mathrm{AU}$. While we have insufficient statistics to place strong constraints on the power law of index -1, we can rule out the other two with increasing confidence as larger values of the upper limit are considered. For example, a power law of the form $\frac{d N}{d a} \propto a^{-0.25}$ must cut-off at $\sim 23 \mathrm{AU}$ (95\% confidence), while the most likely power law of index -0.61 must have its cut-off at $70 \mathrm{AU}$ (also at the 95\% confidence level). 


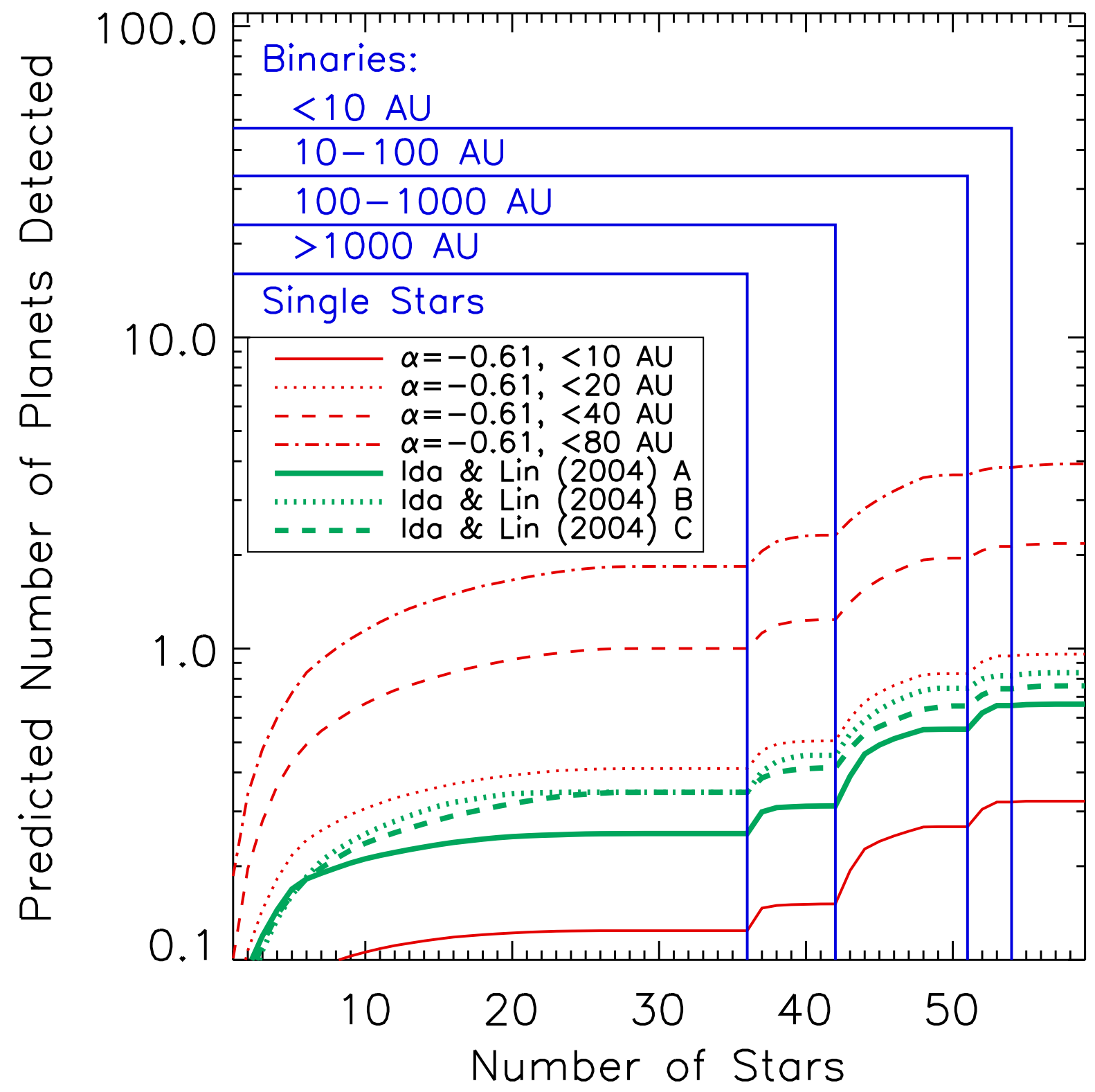

Fig. 17.- The number of planets we would expect to detect at the end of the survey, as a function of the number of target stars observed, out of our total sample of 59. Stars are divided into bins based on binarity, and within each bin the stars are arranged so that the best targets are observed first. The first four models use power laws with $\frac{d N}{d a} \propto a^{-0.61}$, with the upper cut-off given. These models can be ruled out with increasing confidence with cutoffs beyond $40 \mathrm{AU}$ as increasingly close binaries are added to the sample. Since the three Ida \& Lin (2004) models predict less than one planet from our survey, we can only place very limited constraints on these models at this time, namely that cases $\mathrm{A}, \mathrm{B}$, and $\mathrm{C}$ are inconsistent with our null result at the $48 \%, 57 \%$, and $53 \%$ confidence levels, respectively, if all binaries are included in our sample. 
Table 1. Target Stars

\begin{tabular}{|c|c|c|c|c|c|c|c|c|c|}
\hline Target & $\mathrm{RA}^{1}$ & $\operatorname{Dec}^{1}$ & Distance $(\mathrm{pc})^{2}$ & Sp. Type & Age (Myr) & $\mathrm{V}^{1}$ & $\mathrm{H}^{3}$ & $\mathrm{Ks}^{3}$ & Obs. Mode \\
\hline \multicolumn{10}{|l|}{ Biller et al. (2007) } \\
\hline HIP 1481 & $\begin{array}{llll}00 & 18 & 26.1\end{array}$ & -632839.0 & 40.95 & F8/G0V & 30 & 7.46 & 6.25 & 6.15 & VLT SDI \\
\hline HD 8558 & 012321.2 & $\begin{array}{lll}-57 & 28 & 50.7\end{array}$ & 49.29 & G6V & 30 & 8.54 & 6.95 & 6.85 & VLT SDI \\
\hline HD 9054 & $\begin{array}{lll}01 & 28 & 08.7\end{array}$ & $\begin{array}{lll}-52 & 38 & 19.2\end{array}$ & 37.15 & $\mathrm{~K} 1 \mathrm{~V}$ & 30 & 9.35 & 6.94 & 6.83 & VLT SDI \\
\hline HIP 9141 & 015748.9 & -215405.0 & 42.35 & G3/G5V & 30 & 8.11 & 6.55 & 6.47 & VLT SDI \\
\hline $\mathrm{BD}+05378$ & 024125.9 & +055918.4 & 40.54 & Mo & 12 & 10.20 & 7.23 & 7.07 & VLT SDI \\
\hline HD 17925 & 025232.1 & -124611.0 & 10.38 & $\mathrm{~K} 1 \mathrm{~V}$ & 115 & 6.05 & 4.23 & 4.17 & VLT SDI \\
\hline Eps Eri & 033255.8 & $\begin{array}{lll}-09 & 27 & 29.7\end{array}$ & 3.22 & $\mathrm{~K} 2 \mathrm{~V}$ & 800 & 3.73 & 1.88 & 1.78 & VLT SDI \\
\hline V577 Per A & 033313.5 & +461526.5 & 33.77 & $\mathrm{G} 5 \mathrm{IV} / \mathrm{V}$ & 70 & 8.35 & 6.46 & 6.37 & MMT SDI \\
\hline GJ 174 & 044118.9 & +205405.4 & 13.49 & K3V & 160 & 7.98 & 5.31 & 5.15 & VLT SDI \\
\hline GJ 182 & 045934.8 & +014700.7 & 26.67 & $\mathrm{M} 1 \mathrm{Ve}$ & 12 & 10.10 & 6.45 & 6.26 & VLT SDI \\
\hline HIP 23309 & $\begin{array}{lll}05 & 00 & 47.1\end{array}$ & $-57 \quad 1525.5$ & 26.26 & $\mathrm{Mo} / 1$ & 12 & 10.09 & 6.43 & 6.24 & VLT SDI \\
\hline AB Dor & 052844.8 & -652654.9 & 14.94 & K1III & 70 & 6.93 & 4.84 & 4.69 & VLT SDI \\
\hline GJ 207.1 & 053344.8 & +01 5643.4 & 16.82 & $\mathrm{M} 2.5 \mathrm{e}$ & 100 & 9.50 & 7.15 & 6.86 & VLT SDI \\
\hline UY Pic & 053656.8 & -475752.9 & 23.87 & KOV & 70 & 7.95 & 5.93 & 5.81 & VLT SDI \\
\hline HIP 30030 & $\begin{array}{lll}06 & 19 & 08.1\end{array}$ & -032620.0 & 52.36 & G0V & 30 & 8.00 & 6.59 & 6.55 & MMT SDI \\
\hline HIP 30034 & $\begin{array}{lll}06 & 19 & 12.9\end{array}$ & $\begin{array}{lll}-58 & 03 & 16.0\end{array}$ & 45.52 & $\mathrm{~K} 2 \mathrm{~V}$ & 30 & 9.10 & 7.09 & 6.98 & VLT SDI \\
\hline HD 45270 & 062230.9 & $\begin{array}{lll}-60 & 13 & 07.1\end{array}$ & 23.50 & G1V & 70 & 6.50 & 5.16 & 5.05 & VLT SDI \\
\hline HD $48189 \mathrm{~A}$ & $\begin{array}{lll}06 & 38 & 00.4\end{array}$ & $\begin{array}{lll}-61 & 32 & 00.2\end{array}$ & 21.67 & $\mathrm{G} 1 / \mathrm{G} 2 \mathrm{~V}$ & 70 & 6.15 & 4.75 & 4.54 & VLT SDI \\
\hline pi01 UMa & $\begin{array}{lll}08 & 39 & 11.7\end{array}$ & +650115.3 & 14.27 & G1.5V & 210 & 5.63 & 4.28 & 4.17 & MMT SDI \\
\hline HD 81040 & 092347.1 & +202152.0 & 32.56 & G0V & 2500 & 7.74 & 6.27 & 6.16 & MMT SDI \\
\hline LQ Hya & 093225.6 & $-11 \quad 1104.7$ & 18.34 & $\mathrm{KOV}$ & 13 & 7.82 & 5.60 & 5.45 & VLT SDI \\
\hline DX Leo & 093243.7 & +265918.7 & 17.75 & KOV & 115 & 7.01 & 5.24 & 5.12 & VLT SDI \\
\hline HD 92945 & 104328.3 & $\begin{array}{llll}-29 & 03 & 51.4\end{array}$ & 21.57 & $\mathrm{~K} 1 \mathrm{~V}$ & 70 & 7.76 & 5.77 & 5.66 & VLT SDI \\
\hline GJ 417 & $\begin{array}{lll}11 & 12 & 32.4\end{array}$ & +354850.7 & 21.72 & G0V & 115 & 6.41 & 5.02 & 4.96 & MMT SDI \\
\hline TWA 14 & 111326.5 & -452343.0 & $46.00^{4}$ & M0 & 10 & 13.00 & 8.73 & 8.49 & VLT SDI \\
\hline TWA 25 & 121530.8 & -394842.0 & $44.00^{4}$ & Mo & 10 & 11.40 & 7.50 & 7.31 & VLT SDI \\
\hline RXJ1224.8-7503 & 122447.3 & $\begin{array}{lll}-75 & 03 & 09.4\end{array}$ & 24.17 & $\mathrm{~K} 2$ & 16 & 10.51 & 7.84 & 7.71 & VLT SDI \\
\hline HD 114613 & 131203.2 & $\begin{array}{lll}-37 & 48 & 10.9\end{array}$ & 20.48 & G3V & 4200 & 4.85 & 3.35 & 3.30 & VLT SDI \\
\hline HD 128311 & 143600.6 & +094447.5 & 16.57 & K0 & 630 & 7.51 & 5.30 & 5.14 & MMT SDI \\
\hline EK Dra & 143900.2 & +641730.0 & 33.94 & G0 & 70 & 7.60 & 6.01 & 5.91 & MMT SDI \\
\hline HD 135363 & $\begin{array}{lll}15 & 07 & 56.3\end{array}$ & $+76 \quad 1202.7$ & 29.44 & G5V & 3 & 8.72 & 6.33 & 6.19 & MMT SDI \\
\hline KW Lup & 154547.6 & -302055.7 & 40.92 & $\mathrm{~K} 2 \mathrm{~V}$ & 2 & 9.37 & 6.64 & 6.46 & VLT SDI \\
\hline HD 155555 AB & 171725.5 & -665704.0 & 30.03 & G5IV & 12 & 7.20 & 4.91 & 4.70 & VLT SDI \\
\hline HD $155555 \mathrm{C}$ & 171727.7 & -665700.0 & 30.03 & M4.5 & 12 & 12.70 & 7.92 & 7.63 & VLT SDI \\
\hline HD 166435 & $\begin{array}{lll}18 & 09 & 21.4\end{array}$ & +295706.2 & 25.24 & G0 & 100 & 6.85 & 5.39 & 5.32 & MMT SDI \\
\hline HD $172555 \mathrm{~A}$ & 184526.9 & -645216.5 & 29.23 & $\mathrm{~A} 5 \mathrm{IV} / \mathrm{V}$ & 12 & 4.80 & 4.25 & 4.30 & VLT SDI \\
\hline CD -64 1208 & 184537.0 & -645144.6 & 34.21 & K7 & 12 & 10.12 & 6.32 & 6.10 & VLT SDI \\
\hline HD 181321 & 192129.8 & -345900.5 & 20.86 & $\mathrm{G} 1 / \mathrm{G} 2 \mathrm{~V}$ & 160 & 6.48 & 5.05 & 4.93 & VLT SDI \\
\hline HD 186704 & 194557.3 & +041454.6 & 30.26 & G0 & 200 & 7.03 & 5.62 & 5.52 & MMT SDI \\
\hline GJ 799B & 204151.1 & -322609.0 & 10.22 & $\mathrm{M} 4.5 \mathrm{e}$ & 12 & 11.00 & 0.00 & -99.00 & VLT SDI \\
\hline GJ 799A & 204151.2 & $\begin{array}{lll}-32 & 26 & 06.6\end{array}$ & 10.22 & M4.5e & 12 & 10.25 & 5.20 & 4.94 & VLT SDI \\
\hline GJ 803 & 204509.5 & $\begin{array}{lll}-3120 & 27.1\end{array}$ & 9.94 & MOVe & 12 & 8.81 & 4.83 & 4.53 & VLT SDI \\
\hline HD 201091 & 210653.9 & +384457.9 & 3.48 & $\mathrm{~K} 5 \mathrm{Ve}$ & 2000 & 5.21 & 2.54 & 2.25 & MMT SDI \\
\hline Eps Indi A & 220321.7 & $\begin{array}{lll}-56 & 47 & 09.5\end{array}$ & 3.63 & $\mathrm{~K} 5 \mathrm{Ve}$ & 1300 & 4.69 & 2.35 & 2.24 & VLT SDI \\
\hline GJ 862 & 222915.2 & $\begin{array}{lll}-30 & 01 & 06.4\end{array}$ & 15.45 & K5V & 6300 & 7.65 & 5.28 & 5.11 & VLT SDI \\
\hline HIP $112312 \mathrm{~A}$ & 224457.8 & -331501.0 & 23.61 & M4e & 12 & 12.20 & 7.15 & 6.93 & VLT SDI \\
\hline HD 224228 & $\begin{array}{lll}23 & 56 & 10.7 \\
\end{array}$ & $\begin{array}{lll}-39 & 03 & 08.4 \\
\end{array}$ & 22.08 & $\mathrm{~K} 3 \mathrm{~V}$ & 70 & 8.20 & 6.01 & 5.91 & VLT SDI \\
\hline \multicolumn{10}{|c|}{ Masciadri et al. (2005) } \\
\hline HIP 2729 & 003451.2 & -615458 & 45.91 & $\mathrm{~K} 5 \mathrm{~V}$ & 30 & 9.56 & 6.72 & 6.53 & VLT Ks \\
\hline AO Men & $06 \quad 1828.2$ & $\begin{array}{lll}-72 & 02 & 42\end{array}$ & 38.48 & $\mathrm{~K} 6 / 7$ & 30 & 10.09 & 6.98 & 6.81 & VLT Ks \\
\hline $\mathrm{BD}+21729$ & 073923.0 & 020101 & 14.87 & K7 & 30 & 9.82 & 6.09 & 5.87 & VLT H \\
\hline TWA 6 & 101828.8 & $\begin{array}{lll}-31 & 50 & 02\end{array}$ & $77.00^{4}$ & $\mathrm{~K} 7$ & 30 & 11.62 & 8.18 & 8.04 & VLT Ks \\
\hline $\mathrm{BD}+12447$ & 102855.5 & 005028 & 7.23 & M2 & 12 & 9.63 & 5.61 & 5.31 & VLT H \\
\hline TWA 8A & 113241.5 & -265155 & $21.00^{4}$ & M2 & 115 & 12.10 & 7.66 & 7.43 & VLT Ks \\
\hline TWA 8B & 113241.5 & -265155 & $21.00^{4}$ & M5 & 100 & 15.20 & 9.28 & 9.01 & VLT Ks \\
\hline TWA 9A & 114824.2 & $\begin{array}{lll}-37 & 28 & 49\end{array}$ & 50.33 & K5 & 800 & 11.26 & 8.03 & 7.85 & VLT Ks \\
\hline TWA 9B & 114824.2 & $\begin{array}{lll}-37 & 28 & 49\end{array}$ & 50.33 & M1 & 70 & 14.10 & 9.38 & 9.15 & VLT Ks \\
\hline SAO 252852 & 144228.1 & -645843 & $16.40^{5}$ & $\mathrm{~K} 5 \mathrm{~V}$ & 160 & 8.47 & 5.69 & 5.51 & VLT H \\
\hline V343 Nor & 153857.6 & $\begin{array}{lll}-57 & 42 & 27\end{array}$ & 39.76 & K0V & 12 & 8.14 & 5.99 & 5.85 & VLT Ks \\
\hline PZ Tel & 185305.9 & -501050 & 49.65 & $\mathrm{~K} 0 \mathrm{Vp}$ & 12 & 8.42 & 6.49 & 6.37 & VLT Ks \\
\hline BD-17 6128 & $\begin{array}{lll}20 & 56 & 02.7\end{array}$ & $\begin{array}{lll}-17 & 10 & 54\end{array}$ & 47.70 & K7 & 70 & 10.60 & 7.25 & 7.04 & VLT Ks \\
\hline
\end{tabular}


${ }^{4}$ Distance from Song et al. (2003)

${ }^{5}$ Distance from Zuckerman et al. (2001a) 
Table 2. Age Determination for Target Stars

\begin{tabular}{|c|c|c|c|c|c|c|c|c|}
\hline Target & Sp. Type* & Li EW (mas)* & Li Age (Myr) & $\mathrm{R}^{\prime}{ }_{H K}^{*}$ & $\mathrm{R}_{H K}^{\prime}$ Age & Group Membership $^{1}$ & Group Age $^{1}$ & Adopted Age \\
\hline \multicolumn{9}{|c|}{ Biller et al. (2007) } \\
\hline HIP 1481 & F8/G0V ${ }^{2}$ & $129^{3}$ & 100 & $-4.360^{4}$ & 200 & Tuc/Hor & 30 & 30 \\
\hline HD 8558 & $\mathrm{G} 6 \mathrm{~V}^{2}$ & $205^{5}$ & 13 & & & Tuc/Hor & 30 & 30 \\
\hline HD 9054 & $\mathrm{~K} 1 \mathrm{~V}^{2}$ & $170^{5}$ & 160 & $-4.236^{6}$ & $<100$ & Tuc/Hor & 30 & 30 \\
\hline HIP 9141 & $\mathrm{G} 3 / \mathrm{G} 5 \mathrm{~V}^{7}$ & $181^{8}$ & 13 & & & Tuc/Hor & 30 & 30 \\
\hline $\mathrm{BD}+05378$ & $\mathrm{MO}^{9}$ & $15^{10}$ & & & & $\beta \mathrm{Pic}$ & 12 & 12 \\
\hline HD 17925 & $\mathrm{~K} 1 \mathrm{~V}^{7}$ & $194^{8}$ & 50 & $-4.357^{6}$ & 200 & Her/Lyr & 115 & 115 \\
\hline Eps Eri & $\mathrm{K}_{2} \mathrm{~V}^{11}$ & & 0 & $-4.598^{6}$ & 1300 & 101 & 10 & $800^{12}$ \\
\hline V577 Per A & $\mathrm{G} 5 \mathrm{IV} / \mathrm{V}^{13}$ & $219^{13}$ & 3 & & & AB Dor & 70 & 70 \\
\hline GJ 174 & $\mathrm{~K}^{3} \mathrm{~V}^{14}$ & $118^{8}$ & 160 & $-4.066^{6}$ & $<100$ & & & 160 \\
\hline GJ 182 & $\mathrm{M} 1 \mathrm{Ve}^{10}$ & $280^{15}$ & 12 & & & & & 12 \\
\hline AB Dor & $\mathrm{K}_{1 \mathrm{III}}{ }^{2}$ & $267^{8}$ & 10 & $-3.880^{6}$ & $<100$ & AB Dor & 70 & 70 \\
\hline GJ 207.1 & $\mathrm{M} 2.5 \mathrm{e}^{16}$ & & & & & & & $100^{17}$ \\
\hline HIP 23309 & $\mathrm{M} 0 / 1^{18}$ & $294^{18}$ & 12 & $-3.893^{6}$ & & $\beta$ Pic & 12 & 12 \\
\hline UY Pic & $\mathrm{KOV}^{19}$ & $263^{8}$ & 10 & $-4.234^{6}$ & $<100$ & AB Dor & 70 & 70 \\
\hline HD 45270 & $\mathrm{G} 1 \mathrm{~V}^{2}$ & $149^{5}$ & & & & AB Dor & 70 & 70 \\
\hline HD 48189 A & $\mathrm{G} 1 / \mathrm{G} 2 \mathrm{~V}^{2}$ & $145^{8}$ & 25 & $-4.268^{6}$ & 100 & AB Dor & 70 & 70 \\
\hline HIP 30030 & $\mathrm{GOV}^{20}$ & $219^{8}$ & 2 & & & Tuc/Hor & 30 & 30 \\
\hline HIP 30034 & $\mathrm{~K} 2 \mathrm{~V}^{2}$ & & & & & Tuc/Hor & 30 & 30 \\
\hline pi01 UMa & $\mathrm{G} 1.5 \mathrm{~V}^{21}$ & $135^{8}$ & 100 & $-4.400^{22}$ & 320 & & & 210 \\
\hline DX Leo & $\mathrm{K}_{0} \mathrm{~V}^{21}$ & $180^{8}$ & 100 & $-4.234^{6}$ & $<100$ & Her /Lyr & 115 & 115 \\
\hline HD 81040 & G0V 21 & $24^{23}$ & 2500 & & & & & 2500 \\
\hline LQ Hya & $\mathrm{KOV}^{21}$ & $247^{8}$ & 13 & & & & & 13 \\
\hline HD 92945 & $\mathrm{~K} 1 \mathrm{~V}^{21}$ & $138^{8}$ & 160 & $-4.393^{6}$ & 320 & AB Dor & 70 & 70 \\
\hline GJ 417 & GoV 24 & $76^{25}$ & 250 & $-4.368^{26}$ & 250 & Her/Lyr & 115 & 115 \\
\hline TWA 14 & $\mathrm{MO}^{27}$ & $600^{27}$ & 8 & & & TW Hya & 10 & 10 \\
\hline RXJ1224.8-7503 & $\mathrm{K} 2^{28}$ & $250^{28}$ & 16 & & & & & 16 \\
\hline TWA 25 & $\mathrm{Mo}^{9}$ & $494^{29}$ & 10 & & & TW Hya & 10 & 10 \\
\hline HD 114613 & G3V $V^{30}$ & $100^{31}$ & 400 & $-5.118^{6}$ & 7900 & & & 4200 \\
\hline EK Dra & $\mathrm{G0}^{32}$ & $212^{8}$ & 2 & $-4.180^{22}$ & $<100$ & AB Dor & 70 & 70 \\
\hline HD 128311 & $\mathrm{KO}^{21}$ & & & $-4.489^{26}$ & 630 & & & 630 \\
\hline HD 135363 & G5V $\mathrm{V}^{21}$ & $220^{8}$ & 3 & & & & & 3 \\
\hline KW Lup & $\mathrm{K} 2 \mathrm{~V}^{30}$ & $430^{33}$ & 2 & & & & & 2 \\
\hline $\mathrm{HD} 155555 \mathrm{AB}$ & $\mathrm{G}^{2} \mathrm{IV}^{18}$ & $205^{8}$ & 6 & $-3.965^{6}$ & $<100$ & $\beta \mathrm{Pic}$ & 12 & 12 \\
\hline HD $155555 \mathrm{C}$ & $\mathrm{M} 4.5^{18}$ & & & & & $\beta \mathrm{Pic}$ & 12 & 12 \\
\hline CD -64 1208 & $\mathrm{~K} 7^{18}$ & $580^{18}$ & 5 & & & $\beta \mathrm{Pic}$ & 12 & 12 \\
\hline HD 166435 & $\mathrm{GO}^{34}$ & & & $-4.270^{22}$ & 100 & & & 100 \\
\hline HD $172555 \mathrm{~A}$ & $\mathrm{~A} 5 \mathrm{IV} / \mathrm{V}^{2}$ & & & & & $\beta \mathrm{Pic}$ & 12 & 12 \\
\hline HD 181321 & $\mathrm{G} 1 / \mathrm{G} 2 \mathrm{~V}^{30}$ & $131^{8}$ & 79 & $-4.372^{6}$ & 250 & & & 160 \\
\hline HD 186704 & $\mathrm{GO}^{35}$ & & & $-4.350^{22}$ & 200 & & & 200 \\
\hline GJ $799 \mathrm{~A}$ & $\mathrm{M} 4.5 \mathrm{e}^{16}$ & & & & & $\beta \mathrm{Pic}$ & 12 & 12 \\
\hline GJ $799 B$ & $\mathrm{M} 4.5 \mathrm{e}^{16}$ & & & & & $\beta \mathrm{Pic}$ & 12 & 12 \\
\hline GJ 803 & $\mathrm{M} 0 \mathrm{Ve}^{16}$ & $51^{8}$ & 30 & & & $\beta \mathrm{Pic}$ & 12 & 12 \\
\hline HD 201091 & $\mathrm{~K} 5 \mathrm{Ve}^{16}$ & & & $-4.704^{6}$ & $2000^{+}$ & & & 2000 \\
\hline Eps Indi A & $\mathrm{K} 5 \mathrm{Ve}^{16}$ & & & $-4.851^{6}$ & 4000 & & & $1300^{36}$ \\
\hline GJ 862 & $\mathrm{~K} 5 \mathrm{~V}^{16}$ & $5^{15}$ & & $-4.983^{6}$ & $6300^{+}$ & & & 6300 \\
\hline HIP $112312 \mathrm{~A}$ & $\mathrm{M} 4 \mathrm{e}^{9}$ & & & & & $\beta \mathrm{Pic}$ & 12 & 12 \\
\hline HD 224228 & $\mathrm{~K}_{3} \mathrm{~V}^{30}$ & $53^{8}$ & 630 & $-4.468^{6}$ & 500 & AB Dor & 70 & 70 \\
\hline \multicolumn{9}{|c|}{ Masciadri et al. (2005) } \\
\hline HIP 2729 & $\mathrm{~K} 5 \mathrm{~V}^{2}$ & & & & & Tuc/Hor & 30 & 30 \\
\hline AO Men & $\mathrm{K} 6 / 7^{18}$ & $357^{18}$ & 6 & $-3.755^{6}$ & & $\beta \mathrm{Pic}$ & 12 & 12 \\
\hline $\mathrm{BD}+21729$ & $\mathrm{~K} 7^{21}$ & & & & & Her/Lyr & 115 & 115 \\
\hline TWA 6 & $\mathrm{~K} 7^{37}$ & $560^{37}$ & 3 & & & TW Hya & 10 & 10 \\
\hline $\mathrm{BD}+12447$ & $\mathrm{M} 2^{38}$ & & & & & TW Hya & 150 & 150 \\
\hline TWA $8 \mathrm{~A}$ & $\mathrm{M} 2^{37}$ & $530^{37}$ & 3 & & & TW Hya & 10 & 10 \\
\hline TWA $8 B$ & $\mathrm{M} 5^{37}$ & $560^{37}$ & 3 & & & TW Hya & 10 & 10 \\
\hline TWA $9 \mathrm{~A}$ & $\mathrm{~K} 5^{37}$ & $460^{37}$ & 3 & & & TW Hya & 10 & 10 \\
\hline TWA 9B & $\mathrm{M1}^{37}$ & $480^{37}$ & 3 & & & TW Hya & 10 & 10 \\
\hline SAO 252852 & $\mathrm{~K} 5 \mathrm{~V}^{39}$ & & & & & Her/Lyr & 115 & 115 \\
\hline V343 Nor & $\mathrm{KOV}^{2}$ & $300^{31}$ & 5 & & & $\beta \mathrm{Pic}$ & 12 & 12 \\
\hline PZ Tel & $\mathrm{K} O \mathrm{Vp}^{19}$ & $267^{40}$ & 20 & & & $\beta \mathrm{Pic}$ & 12 & 12 \\
\hline BD-17 6128 & $\mathrm{~K} 7^{41}$ & $400^{42}$ & 3 & & & $\beta \mathrm{Pic}$ & 12 & 12 \\
\hline
\end{tabular}

${ }^{1}$ Group Membership for TWA, $\beta$ Pic, Tuc/Hor, and AB Dor from Zuckerman \& Song (2004), Her/Lyr from López-Santiago et al. (2006). Group Ages from Zuckerman \& Song (2004) (TWA, $\beta$ Pic, and Tuc/Hor), Nielsen et al. (2005) (AB Dor), and López-Santiago et al. (2006) (Her/Lyr)

*Measurement References: 2: Houk \& Cowley (1975), 3: Waite et al. (2005), 4: Henry et al. (1996), 5: Torres et al. (2000), 6: Gray et al. (2006), 
7: Houk \& Smith-Moore (1988), 8: Wichmann et al. 2003), 9: Zuckerman \& Song (2004), 10: Favata et al. (1995), 11: Cowlev et al. (1967), 12: Benedict et al. (2006), 13: Christian \& Mathioudakis (2002), 14: Leaton \& Pagel (1960), 15: Favata et al. (1997), 16: Gliese \& Jahreiss (1991), 17: Lowrance et al. (2005), 18: Zuckerman et al. (2001a), 19: Houk (1978), 20: Cutispoto et al. (1995), 21: Montes et al. (2001), 22: Wright et al. (2004), 23: Sozzetti et al. (2006), 24: Bidelman (1951), 25: Gaidos et al. (2000), 26: Grav et al. (2003), 27: Zuckerman et al. (2001b), 28: Alcala et al. (1995), 29: Song et al. (2003), 30: Houk (1982), 31: Randich et al. (1993), 32: Gliese \& Jahreiß (1979), 33: Neuhauser \& Brandner (1998), 34: Eggen (1996), 35: Abt (1985), 36: Lachaume et al. (1999), 37: Webb et al. (1999), 38: Vyssotsky et al. (1946), 39: Evans (1961), 40: Soderblom et al. (1998), 41: Nesterov et al. (1995), 42: Mathioudakis et al. (1995)

${ }^{+}$In general, we have only determined Ca R' ${ }_{H K}$ ages for stars with spectral types $\mathrm{K} 1$ or earlier, but in the case of these two K5 stars, we have only the $\mathrm{R}_{H K}^{\prime}$ measurement on which to rely for age determination. The calibration of Mt. Wilson S-index to R' $H K$ for K5 stars (B-V $\sim 1.1$ mag) has not been well-defined (Noyes et al. (1984); specifically the photospheric subtraction), and hence applying a $\mathrm{R}^{\prime} H K$ vs. age relation for K5 stars is unlikely to yield useful ages. Although we adopt specific values for the ages of these stars, it would be more accurate to state simply that these stars have ages $>1$ Gyr. As a result, almost all simulated planets are too faint to detect around these stars, so the precise error in the age does not significantly affect our final results. 
Table 3. Binaries

\begin{tabular}{|c|c|c|c|c|}
\hline Target & Sep (") & Sep. $(A U)$ & Reference & Companion Type \\
\hline \multicolumn{5}{|c|}{ Biller et al. (2007) } \\
\hline HIP 9141 & 0.15 & 6.38 & Biller et al. (2007) & \\
\hline V577 Per A & 7 & 230 & Pounds et al. (1993) & M0 \\
\hline AB Dor & $9(\mathrm{Ba} / \mathrm{Bb})$ & $134(\mathrm{Ba} / \mathrm{Bb})$ & Close et al. (2005) & Binary $M$ stars \\
\hline AB Dor & $0.15(\mathrm{C})$ & $2.24(\mathrm{C})$ & Close et al. (2005) & Very low-mass M Star \\
\hline HIP 30034 & 5.5 & 250 & Chauvin et al. (2005) & Planet/Brown Dwarf \\
\hline HD $48189 \mathrm{~A}$ & $0.76(\mathrm{~B})$ & 16.5 & Fabricius \& Makarov (2000) & K star \\
\hline HD $48189 \mathrm{~A}$ & 0.14 & 3.03 & Biller et al. (2007) & \\
\hline DX Leo & 65 & 1200 & Lowrance et al. (2005) & M5.5 \\
\hline EK Dra & SB & SB & Metchev \& Hillenbrand (2004) & M2 \\
\hline HD 135363 & 0.26 & 7.65 & Biller et al. (2007) & \\
\hline HD $155555 \mathrm{AB}$ & $\mathrm{SB}(\mathrm{AB})$ & $\mathrm{SB}(\mathrm{AB})$ & Bennett et al. (1967) & G5 and K0 SB \\
\hline HD $155555 \mathrm{AB}$ & $18(\mathrm{C})$ & $1060(\mathrm{C})$ & Zuckerman et al. (2001a) & Target Star $155555 \mathrm{C}, \mathrm{M} 4.5$ \\
\hline HD 172555 A & 71 & 2100 & Simon \& Drake (1993) & Target Star CD -64 1208, K7 \\
\hline HD 186704 & 13 & 380 & Aitken \& Doolittle (1932) & \\
\hline GJ 799A & 3.6 & 36 & Wilson $(1954)$ & Target Star GJ 799B, M4.5 \\
\hline HD 201091 & 16 & 55 & Baize $(1950)$ & K5 \\
\hline Eps Indi A & 400 & 1500 & McCaughrean et al. (2004) & Binary Brown Dwarf \\
\hline HIP 112312 & 100 & 2400 & Song et al. (2002) & $\mathrm{M} 4.5$ \\
\hline \multicolumn{5}{|c|}{ Masciadri et al. (2005) } \\
\hline TWA 8A & 13 & 270 & Jayawardhana et al. (1999) & Target Star TWA 8B, M5 \\
\hline TWA 9A & 9 & 576 & Jayawardhana et al. (1999) & Target Star TWA 9B, M1 \\
\hline SAO 252852 & 15.7 & 260 & Poveda et al. (1994) & HD 128898, Ap \\
\hline V343 Nor & 10 & 432 & Song et al. (2003) & M4.5 \\
\hline BD-17 6128 & 2 & 100 & Neuhäuser et al. (2002) & M2 \\
\hline
\end{tabular}

